TRANSACTIONS OF THE

AMERICAN MATHEMATICAL SOCIETY

Volume 358, Number 11, November 2006, Pages 5083-5117

S 0002-9947(06)04019-0

Article electronically published on June 20, 2006

\title{
FREQUENTLY HYPERCYCLIC OPERATORS
}

\author{
FRÉDÉRIC BAYART AND SOPHIE GRIVAUX
}

\begin{abstract}
We investigate the subject of linear dynamics by studying the notion of frequent hypercyclicity for bounded operators $T$ on separable complex $\mathcal{F}$-spaces: $T$ is frequently hypercyclic if there exists a vector $x$ such that for every nonempty open subset $U$ of $X$, the set of integers $n$ such that $T^{n} x$ belongs to $U$ has positive lower density. We give several criteria for frequent hypercyclicity, and this leads us in particular to study linear transformations from the point of view of ergodic theory. Several other topics which are classical in hypercyclicity theory are also investigated in the frequent hypercyclicity setting.
\end{abstract}

\section{INTRODUCTION}

1.1. The global setting. In this paper, we will be concerned with the study of the dynamics of linear operators on a complex separable $\mathcal{F}$-space $X$, i.e. a topological space whose topology is induced by a complete invariant metric $\rho$. Let us first recall the following definitions: a bounded operator $T$ on $X$ is said to be topologically transitive if for every pair $(U, V)$ of nonempty open subsets of $X$ there exists an integer $n$ (and hence infinitely many integers) such that $T^{n}(U) \cap V$ is nonempty. Since the space $X$ is assumed to be separable, a simple Baire Category argument shows that $T$ is topologically transitive if and only if there exists a vector $x$ of $X$ such that the orbit $\mathcal{O} r b(x, T)=\left\{T^{n} x ; n \geq 0\right\}$ of $x$ under the action of $T$ is dense in $X$. Such a vector is called a hypercyclic vector for $T$, and $T$ itself is called a hypercyclic operator. We will denote by $H C(T)$ the set of hypercyclic vectors for $T$.

Studying the dynamics of an operator is studying the behaviour of the iterates $T^{n} x$ for $x \in X$. One may think at first sight that the dynamics of a linear operator ought to be less complicated than the dynamics of a general continuous function on a metric space. When the space $X$ is finite dimensional, this proves to be true: no operator on $X$ is ever hypercyclic, and using Jordan canonical forms, one can easily describe the behaviour of all the orbits. But the situation in infinite-dimensional spaces is more involved, as the following result of Feldman [19] testifies: there exists a hypercyclic operator such that each continuous function on a compact

Received by the editors April 15, 2004 and, in revised form, November 25, 2004.

2000 Mathematics Subject Classification. Primary 47A16, 47A35, 37B05, 37A05.

Key words and phrases. Hypercyclic operators, frequently hypercyclic operators, unimodular point spectrum, ergodic and weak-mixing measure-preserving linear transformations, Gaussian measures on Hilbert spaces, Fock spaces.

This work was supported in part by the European Community's Human Potential Programme under contract HPRN-CT-2000-00116 (Analysis and Operators). 
metric space $X$ is topologically conjugate to the restriction of this operator to an invariant compact set.

The study of hypercyclic operators originated in the work of Birkhoff in 1929 ([11]), who proved that the translation operator $f \mapsto[z \mapsto f(z+1)]$ on $\mathcal{H}(\mathbb{C})$ is hypercyclic. The first example of a hypercyclic operator in the Banach space setting dates back to 1969 (46]), and is due to Rolewicz. Hypercyclic operators have been extensively studied since the 80's, one reason for the interest in this topic being the relation of hypercyclicity to the Invariant Subset Problem. For a thorough account of what has been done in this area, see the survey [32], and also 33] for more recent results. Our purpose in this paper is to study hypercyclic operators from the point of view of topological dynamics and ergodic theory, and to quantify the frequency with which the iterates of a given hypercyclic vector visit any nonempty open set. We recall the following definition.

Definition 1.1. Let $A$ be a subset of $\mathbb{N}$. We say that $A$ has positive lower density if

$$
\underline{\operatorname{dens}}(A)=\liminf _{N \rightarrow+\infty} \frac{\#\{n \leq N ; n \in A\}}{N}>0,
$$

where $\# B$ stands for the cardinal number of $B$.

Our main definition is:

Definition 1.2. An operator $T$ on $X$ is said to be frequently hypercyclic provided there exists a vector $x$ such that for every nonempty open subset $U$ of $X$, the set of integers $n$ such that $T^{n} x$ belongs to $U$ has positive lower density. In this case, $x$ is called a frequently hypercyclic vector for $T$, and the set of frequently hypercyclic vectors will be denoted by $F H C(T)$.

It will be convenient in the sequel to reformulate the above definition as follows: for any operator $T$ on an $\mathcal{F}$-space $X$ endowed with a complete invariant metric $\rho$, any two vectors $x$ and $f$ in $X$ and any positive real number $\varepsilon$, consider the following subset of $\mathbb{N}$ :

$$
\operatorname{App}(T, x, f, \varepsilon)=\left\{n \in \mathbb{N} ; \rho\left(T^{n} x, f\right)<\varepsilon\right\} .
$$

Then $T$ is frequently hypercyclic if and only if there exists a vector $x$ such that, for each $f \in X$ and each $\varepsilon>0$, dens $(A p p(T, x, f, \varepsilon))>0$.

1.2. Motivation: the Hypercyclicity Criterion Problem. A well-known open question in hypercyclicity is to determine whether every hypercyclic operator satisfies the so-called Hypercyclicity Criterion (the first version of it was given by Kitai in [38, and it was independently rediscovered and strengthened by Gethner and Shapiro in [26]). The version we give here is due to Bès (see for instance [10]).

Hypercyclicity Criterion. Suppose that $T$ is a bounded operator on $X$ such that there exist a strictly increasing sequence $\left(n_{k}\right)$ of positive integers, two dense subsets $V$ and $W$ of $X$ and a sequence $\left(S_{n_{k}}\right)$ of maps (not necessarily linear nor continuous) $S_{n_{k}}: W \rightarrow X$ such that:

1. for every $v \in V$, the sequence $\left(T^{n_{k}} v\right)_{k \geq 0}$ tends to 0 ,

2. for every $w \in W$, the sequence $\left(S_{n_{k}} w\right)_{k \geq 0}$ tends to 0 ,

3. for every $w \in W$, the sequence $\left(T^{n_{k}} S_{n_{k}} w\right)_{k \geq 0}$ tends to $w$.

Then $T$ is hypercyclic.

It was shown by Bès and Peris in [10 that the Hypercyclicity Criterion Problem is equivalent to the following question of Herrero ([34]). 
Question 1.3. If $T$ is hypercyclic on $X$, is $T \oplus T$ hypercyclic on $X \oplus X$ ?

This is a question of simultaneous approximation, and until now it has been investigated using Baire Category arguments and some "regularity" assumptions (see for instance [10, 31] or [8]). This has meant studying the "global" behaviour of the operator, i.e. the behaviour of the sets $T^{-n}(U), n \geq 0$, where $U$ is a nonempty open set. Here we focus on the study of the "individual" behaviour of an orbit, i.e. of the iterates $T^{n} x, n \geq 0$, for some fixed $x$. This implies forgetting about Baire Category arguments. A natural tool which can replace it is measure theory, and this comes quite naturally into the picture: if $T$ is a measure-preserving transformation on a probability space $(X, \mathcal{B}, \mu)$, it is known that $T \times T$ is ergodic on $(X \times X, \mathcal{B} \otimes \mathcal{B}, \mu \otimes \mu)$ if and only if $T$ is weak-mixing (the definitions are recalled in Section 3). There is an obvious formal similarity between this result and Question 1.3 above, which leads us to search for conditions implying that an operator has an invariant nondegenerate measure with respect to which it is ergodic, weak-mixing, strong-mixing, etc. It turns out that these questions are best investigated in terms of frequent hypercyclicity.

Questions of this kind have already been considered by Flytzanis in 21] and 22, and some related ideas are also present in the work of Bourdon and Shapiro [14. We now give a brief description of our results.

1.3. Main results. At this early stage, it is neither clear whether frequently hypercyclic operators do exist, nor is it clear that a hypercyclic operator is not always frequently hypercyclic. In the first part of this paper (Section 2), we give a criterion which ensures that an operator is frequently hypercyclic (Theorem 2.1). This criterion, which bears a similarity to the Hypercyclicity Criterion, relies on a technical lemma (Lemma 2.2) whose proof is given also in Section 2. In this way Birkhoff's translation operator, MacLane's differentiation's operator on $\mathcal{H}(\mathbb{C})$, and multiples $\omega B,|\omega|>1$, of the backward shift $B$ on $\ell_{p}, 1 \leq p<+\infty$, or $c_{0}$ can be seen to be frequently hypercyclic. We also give some examples of hypercyclic operators which are not frequently hypercyclic (Example 2.9). This is the case for instance for the the backward shift on the Bergman space $A^{2}$.

In Section 3, we restrict ourselves to the Hilbert space setting and show that whenever the operator $T$ has sufficiently many eigenvectors associated to eigenvalues of modulus 1, $T$ is frequently hypercyclic (Theorem 3.2). In particular $T$ is hypercyclic (and even weakly topologically mixing), which can be seen as a counterpart to the Godefroy-Shapiro Criterion for hypercyclicity (27]). The proof of Theorem 3.2 uses tools from ergodic theory, which were introduced in this setting by Flytzanis in his seminal paper [21]: an operator which has a perfectly spanning set of eigenvectors associated to unimodular eigenvalues (Definition 3.1) admits a nondegenerate Gaussian invariant measure with respect to which $T$ is a weak-mixing transformation (Theorem 3.22). In many cases, $T$ is even strong-mixing (Theorem 3.29). From these results follows that quite a lot of hypercyclic operators are in fact frequently hypercyclic: perturbations of operators by big multiples of the backward shift, adjoints of multipliers on holomorphic function spaces, hyperbolic and parabolic composition operators on the Hardy space associated to automorphisms of the unit disk, etc.

In Section 4, we study the structure of the set $F H C(T)$ of frequently hypercyclic vectors of a given operator $T$, focusing on the lack of Baire Category methods which lies at the core of the frequent hypercyclicity theory: all the operators considered in 
Sections 2 and 3 have been proved to be frequently hypercyclic using either a direct construction of a frequently hypercyclic vector or measure-theoretic considerations. These arguments cannot be simplified by a Baire Category argument: for all concrete frequently hypercyclic operators exhibited in this paper, $F H C(T)$ does not contain a residual set (Proposition 4.1). Thus several results which are classical in hypercyclicity theory wax into somewhat puzzling questions when transposed in the frequent hypercyclicity setting. When residuality does not play a crucial part in the proof of a hypercyclicity result, it usually can be extended to a frequent hypercyclicity result. This is the case for instance for a well-known result of Ansari regarding powers of hypercyclic operators ([2]): we prove that if $T$ is frequently hypercyclic, $T^{q}$ is also frequently hypercyclic for any $q \geq 1$, and that $F H C\left(T^{q}\right)=F H C(T)$. On the other hand, frequently hypercyclic operators can behave in an unexpected way when considering problems of common frequent hypercyclicity: if $B$ is the backward shift on $\ell_{2}$ and $|\lambda|>1, \lambda B$ is frequently hypercyclic, and it was proved by Abakumov and Gordon ([1]) that $\bigcap_{|\lambda|>1} H C(\lambda B)$ is nonempty. We show that $\bigcap_{|\lambda|>1} F H C(\lambda B)$ is empty (Theorem 4.5). But if $T_{a}$ is the translation operator $f \mapsto[z \mapsto f(z+a)]$ on $\mathcal{H}(\mathbb{C})$, then $\bigcap_{a \in \mathbb{C} \backslash\{0\}} F H C\left(T_{a}\right)$ is nonempty (Theorem 4.6). Thus the structure of $F H C(T)$ remains rather mysterious, and seems to deserve to be investigated further.

\section{A FREQUENT HYPERCYCLICITY CRITERION}

2.1. Main result. Our first task is to exhibit some examples of frequently hypercyclic operators. This is done thanks to the following Frequent Hypercyclicity Criterion.

Theorem 2.1. Let $X$ be a separable $\mathcal{F}$-space, $\rho$ an invariant metric which makes it complete, and $T$ a continuous operator on $X$. Suppose that there exist a dense sequence $\left(x_{l}\right)_{l \geq 1}$ of vectors of $X$ and a map $S$ defined on $X$ such that

(1) the series $\sum_{k \geq 1} \rho\left(T^{k} x_{l}, 0\right)$ is convergent for every $l \geq 1$,

(2) the series $\sum_{k \geq 1} \rho\left(S^{k} x_{l}, 0\right)$ is convergent for every $l \geq 1$,

(3) $T S=I$.

Then $T$ is frequently hypercyclic.

When $T$ is frequently hypercyclic, it clearly has a dense set of frequently hypercyclic vectors. This criterion is a strengthened version of the Hypercyclicity Criterion, but its proof does not rely on a Baire Category argument. A frequently hypercyclic vector is obtained in a constructive way by making use of the following lemma.

Lemma 2.2. There exist a strictly increasing sequence $\left(n_{k}\right)_{k \geq 1}$ of integers, a sequence $\left(m_{k}\right)_{k \geq 1}$ of integers and a sequence $\left(R_{k}\right)_{k \geq 1}$ of positive real numbers such that:

(1) $n_{k} \geq R_{k}$ and $n_{k+1}-n_{k} \geq R_{k+1}+R_{k}$ for every $k \geq 1$,

(2) for every $l \geq 1$ and $R>0$, the set $E(l, R)=\left\{n_{k} ; R_{k} \geq R\right.$ and $\left.m_{k}=l\right\}$ has positive lower density.

We postpone the proof of this technical lemma, and now give the proof of Theorem 2.1. 
Proof. The proof will be carried out in the Banach space setting, the obvious modifications (using the invariance of the metric $\rho$ ) in the general case being left to the reader. Let $c_{k}(l)=\left\|T^{k} x_{l}\right\|+\left\|S^{k} x_{l}\right\|$. The series $\sum_{k \geq 1} c_{k}(l)$ is convergent for every $l \geq 1$. Let $\left(N_{l}\right)_{l \geq 1}$ be a strictly increasing sequence of integers such that for every $j \leq l$,

$$
\sum_{k \geq N_{j}} c_{k}(l) \leq \frac{1}{2^{j+l}}
$$

Let $\left(n_{k}\right),\left(m_{k}\right)$ and $\left(R_{k}\right)$ be the three sequences given by Lemma 2.2, and set

$$
y_{k}= \begin{cases}x_{m_{k}} & \text { if } R_{k} \geq N_{m_{k}} \\ 0 & \text { otherwise }\end{cases}
$$

We claim that $x$ is a frequently hypercyclic vector, where

$$
x=\sum_{k \geq 1} S^{n_{k}} y_{k} .
$$

Observe first that this series is convergent:

$$
\sum_{k \geq 1}\left\|S^{n_{k}} y_{k}\right\|=\sum_{l \geq 1} \sum_{\substack{m_{k}=l \\ R_{k} \geq N_{l}}}\left\|S^{n_{k}} x_{l}\right\| \leq \sum_{l \geq 1} \sum_{\substack{m_{k}=l \\ R_{k} \geq N_{l}}} c_{n_{k}}(l) .
$$

Since $R_{k} \geq N_{l}, n_{k} \geq N_{l}$, hence

$$
\sum_{\substack{m_{k}=l \\ R_{k} \geq N_{l}}} c_{n_{k}}(l) \leq \sum_{p \geq N_{l}} c_{p}(l) \leq \frac{1}{4^{l}}
$$

which proves that the series $\sum_{k \geq 1} S^{n_{k}} y_{k}$ is convergent. In order to show that $x$ is frequently hypercyclic, let $f$ be a vector in $X$ and $\varepsilon>0$. Let $l_{0}$ be such that $\left\|f-x_{l_{0}}\right\| \leq \frac{\varepsilon}{2}$ and $\frac{4}{2^{l_{0}}} \leq \frac{\varepsilon}{2}$, and choose $R$ such that $R>N_{l_{0}}$. We are going to show that whenever $n$ belongs to $E\left(l_{0}, R\right),\left\|T^{n} x-f\right\| \leq \varepsilon$. Let $n=n_{k_{0}}$ be such an integer. Then

$$
\begin{aligned}
\left\|T^{n} x-f\right\| & \leq\left\|T^{n} x-x_{l_{0}}\right\|+\frac{\varepsilon}{2} \\
& \leq\left\|T^{n_{k_{0}}} S^{n_{k_{0}}} y_{k_{0}}-x_{l_{0}}\right\|+\sum_{k<k_{0}}\left\|T^{n} S^{n_{k}} y_{k}\right\|+\sum_{k>k_{0}}\left\|T^{n} S^{n_{k}} y_{k}\right\|+\frac{\varepsilon}{2} .
\end{aligned}
$$

The first term is equal to $T^{n_{k_{0}}} S^{n_{k_{0}}} x_{m_{k_{0}}}-x_{l_{0}}=x_{l_{0}}-x_{l_{0}}=0$. We now have to obtain an upper bound for the remaining two sums.

- If $k<k_{0}$, then $T^{n} S^{n_{k}} y_{k}=T^{n-n_{k}} y_{k}$. Hence

$$
\begin{aligned}
\sum_{k<k_{0}}\left\|T^{n} S^{n_{k}} y_{k}\right\| & =\sum_{l \geq 1} \sum_{\substack{k<k_{0} \\
m_{k}=l \\
R_{k} \geq N_{l}}}\left\|T^{n-n_{k}} x_{l}\right\| \leq \sum_{l \geq 1} \sum_{\substack{k<k_{0} \\
m_{k}=l \\
R_{k} \geq N_{l}}} c_{n-n_{k}}(l) \\
& \leq \sum_{l=1}^{l_{0}} \sum_{\substack{k<k_{0} \\
m_{k}=l \\
R_{k} \geq N_{l}}} c_{n-n_{k}}(l)+\sum_{l=l_{0}+1}^{+\infty} \sum_{\substack{k<k_{0} \\
m_{k}=l \\
R_{k} \geq N_{l}}} c_{n-n_{k}}(l) .
\end{aligned}
$$


Now $n-n_{k}=n_{k_{0}}-n_{k} \geq R_{k_{0}} \geq N_{l_{0}}$, the final inequality holding because $n_{k_{0}}$ belongs to $E\left(l_{0}, R\right)$ and $R>N_{l_{0}}$; hence,

$$
\sum_{l=1}^{l_{0}} \sum_{\substack{k<k_{0} \\ m_{k}=l \\ R_{k} \geq N_{l}}} c_{n-n_{k}}(l) \leq \sum_{l=1}^{l_{0}} \sum_{p \geq N_{l_{0}}} c_{p}(l) \leq \sum_{l=1}^{l_{0}} \frac{1}{2^{l+l_{0}}} \leq \frac{1}{2^{l_{0}}} \leq \frac{\varepsilon}{8} .
$$

Also, if $k<k_{0}, n-n_{k} \geq n_{k+1}-n_{k} \geq R_{k}$; hence,

$$
\sum_{\substack{l=l_{0}+1 \\ \sum_{k<k_{0}} \\ m_{k}=l \\ R_{k} \geq N_{l}}} c_{n-n_{k}}(l) \leq \sum_{l=l_{0}+1}^{+\infty} \sum_{p \geq N_{l}} c_{p}(l) \leq \sum_{l=l_{0}+1}^{+\infty} \frac{1}{4^{l}} \leq \frac{1}{2^{l_{0}}}<\frac{\varepsilon}{8} .
$$

Thus

$$
\sum_{k<k_{0}}\left\|T^{n} S^{n_{k}} y_{k}\right\|<\frac{\varepsilon}{4}
$$

- It remains to deal with the sum $\sum_{k>k_{0}}\left\|T^{n} S^{n_{k}} y_{k}\right\|$. Here $T^{n} S^{n_{k}} y_{k}=S^{n_{k}-n} x_{l}$ if $m_{k}=l$, and $R_{k} \geq N_{l}$. This gives

$$
\sum_{k>k_{0}}\left\|T^{n} S^{n_{k}} y_{k}\right\| \leq \sum_{l=1}^{l_{0}} \sum_{\substack{k>k_{0} \\ m_{k}=l \\ R_{k} \geq N_{l}}}\left\|S^{n_{k}-n} x_{l}\right\|+\sum_{\substack{l=l_{0}+1 \\+\infty}}^{+\infty}\left\|S^{n_{k}-n} x_{l}\right\| .
$$

Since $n_{k}-n \geq n_{k_{0}+1}-n_{k_{0}} \geq R_{k_{0}} \geq N_{l_{0}}$, the first sum is less than

$$
\sum_{l=1}^{l_{0}} \sum_{p \geq N_{k_{0}}} c_{p}(l) \leq \sum_{l=1}^{l_{0}} \frac{1}{2^{l+l_{0}}} \leq \frac{1}{2^{l_{0}}}<\frac{\varepsilon}{8} .
$$

On the other hand, if $k>k_{0}, n_{k}-n \geq n_{k}-n_{k-1} \geq R_{k}$; hence the second sum is again less than

$$
\sum_{l=l_{0}+1}^{+\infty} \sum_{p \geq N_{l}} c_{p}(l) \leq \sum_{l=l_{0}+1}^{+\infty} \frac{1}{4^{l}} \leq \frac{1}{2^{l_{0}}}<\frac{\varepsilon}{8}
$$

Putting everything together, we obtain that if $n=n_{k_{0}}$ belongs to $E\left(l_{0}, R\right)$, then we have $\left\|T^{n} x-f\right\|<\varepsilon$. Therefore $x$ is a frequently hypercyclic vector for $T$, and this proves Theorem 2.1

2.2. Proof of Lemma 2.2, We now proceed with the proof of Lemma 2.2, Let us first fix a sequence $\left(\alpha_{i}\right)_{i \geq 1}$ of positive real numbers satisfying $0<\alpha_{i}<1$ and $\prod_{i=1}^{+\infty} \alpha_{i} \geq 1 / 2$. The proof of the lemma will be done by induction: in Step $j$, we construct three blocks $\left(n_{k}\right)_{k=\Gamma_{j} \ldots \Gamma_{j+1}-1},\left(R_{k}\right)_{k=\Gamma_{j} \ldots \Gamma_{j+1}-1}$ and $\left(m_{k}\right)_{k=\Gamma_{j} \ldots \Gamma_{j+1}-1}$ out of the three sequences $\left(n_{k}\right),\left(R_{k}\right)$ and $\left(m_{k}\right)$ such that three properties are satisfied. We will repeatedly use the following notation:

Notation. $B_{j}=\left\{n_{\Gamma_{j}}, \ldots, n_{\Gamma_{j+1}-1}\right\}, L_{j}=\max B_{j}$ and $E_{j}(l, R)=E(l, R) \cap B_{j}$.

The three properties which must be satisfied at Step $j$ are stated below. The first one is simply taken out of Lemma 2.2. the meaning and interest of the inequalities of the second and third will be made clear during the proof.

Induction Step.

(1) $n_{k} \geq R_{k}$ and $n_{k+1}-n_{k} \geq R_{k+1}+R_{k}$ for every $k=\Gamma_{j}, \ldots, \Gamma_{j+1}-1$, 
(2) we have

$$
\frac{L_{j}}{L_{j}+(j+1)}>\alpha_{j+1},
$$

(3) for every $l \leq j$ and $0 \leq R \leq \frac{j}{2}$,

$$
\frac{\# E_{j}(l, R)}{L_{j}+(j+2)(j+1)} \geq\left(\prod_{i=1}^{j} \alpha_{i}\right) \delta_{l, R},
$$

where $\delta_{l, R}$ is some positive constant which does not depend on $j$ (for instance

$$
\delta_{l, R}=\frac{1}{L_{q}+(q+1)(q+2)} \frac{1}{\left(\prod_{i=1}^{q} \alpha_{i}\right)}
$$

with $q=\max (l,\lfloor R\rfloor+1)$ will do).

Step 1. Set $R_{1}=1, m_{1}=1$ and choose $n_{1}$ large enough so that

$$
\frac{n_{1}}{n_{1}+2}>\alpha_{2} \text {. }
$$

Set $B_{1}=\left\{n_{1}\right\}$. Observe that for any $R \leq 1 / 2$, \# $E_{1}(1, R)=1$. The numbers $\delta_{1, R}$ are definitively adjusted so that the inequalities of assertion (3) of the induction step are satisfied.

Step $j$. Suppose that the construction has been carried out until Step $j-1$. In order to simplify the notations, we let $B_{j-1}=\left\{n_{u}, \ldots, n_{v}\right\}$ be the set of integers $\left(n_{k}\right)$ exhibited throughout Step $j-1$. In particular, one has $L_{j-1}=n_{v}$. The construction for Step $j$ is split into two parts. Our first task is to allow $l$ to go to $j$ and $R$ to go to $j / 2$ in the inequalities of assertion (3). To this aim, we define

$$
\begin{array}{rlll}
n_{v+1}=n_{v}+j & R_{v+1}=j / 2 & m_{v+1}=1 \\
n_{v+2}=n_{v}+2 j & R_{v+2}=j / 2 & m_{v+2}=2 \\
\vdots & \vdots \quad \vdots & & \\
n_{v+j}=n_{v}+j \times j & R_{v+j}=j / 2 & m_{v+j}=j .
\end{array}
$$

Note that for $l \leq j-1$ and $R \leq(j-1) / 2$, the factor $\delta_{l, R}$ appearing in assertion (3) is already defined. To be sure that assertion (3) remains true after Step $j$ for this range of $l$ and $R$, we have to add as many shifted copies of $B_{j-1}$ as necessary, and we will need assertion (2) of the induction hypothesis. So, let $r>0$, whose precise value will be given later. For $p$ in $\{0, \ldots, r\}$ and $i$ in $\{0, \ldots, v-u\}$, set

$$
\begin{aligned}
& n_{v+j+p(v-u+1)+i+1}=(p+1)\left(n_{v}+j\right)+j^{2}+n_{u+i} \\
& R_{v+j+p(v-u+1)+i+1}=R_{u+i} \\
& m_{v+j+p(v-u+1)+i+1}=m_{u+i} .
\end{aligned}
$$

The constraints on $R_{k}$ and $n_{k}$ of assertion (1) are satisfied: this is obvious if $k$ belongs to $\{v+1, \ldots, v+j\}$, and this follows from the induction hypothesis if $k$ is greater than $v+j$. Let us now explain how to choose $r$. Let $l$ and $R$ be such that $l<j$ and $R<(j-1) / 2$, and write

$$
E_{j-1}(l, R)=\left\{n_{1}^{\prime}, \ldots, n_{w}^{\prime}\right\} .
$$

The induction hypothesis implies that

$$
\frac{w}{n_{v}} \geq\left(\prod_{i=1}^{j-1} \alpha_{i}\right) \delta_{l, R} .
$$


Now, observe that $(p+1)\left(n_{v}+j\right)+j^{2}+n_{i}^{\prime}$ belongs to $E_{j}(l, R)$ for any $p$ in $\{0, \ldots, r\}$ and any $i \in\{1, \ldots, w\}$. This yields that

$$
\begin{aligned}
\frac{\# E_{j}(l, R)}{L_{j}+(j+2)(j+1)} & \geq \frac{(r+1) w}{(r+1)\left(L_{j-1}+j\right)+j^{2}+L_{j-1}+(j+2)(j+1)} \\
& \geq\left(\prod_{i=1}^{j-1} \alpha_{i}\right) \delta_{l, R} \frac{(r+1) L_{j-1}}{K_{j}+(r+2)\left(L_{j-1}+j\right)},
\end{aligned}
$$

where $K_{j}$ is a constant which depends only on $j$. This is where the inequality of assertion (2) comes into the picture. We choose $r$ large enough so that

$$
\frac{(r+1) L_{j-1}}{K_{j}+(r+2)\left(L_{j-1}+j\right)} \geq \alpha_{j} .
$$

This can be done since condition (2) is satisfied at Step $j-1$. But it is also necessary to have $r$ large enough so that

$$
\frac{L_{j}}{L_{j}+(j+1)}=\frac{(r+1)\left(n_{v}+j\right)+j^{2}+n_{v}}{(r+1)\left(n_{v}+j\right)+j^{2}+n_{v}+j+1}>\alpha_{j+1} .
$$

With this assumption, we ensure that the inequality of assertion (2) is true after Step $j$, and therefore we prepare Step $j+1$. If now $l$ and $R$ are such that $l=j$ or $\frac{j-1}{2}<R \leq \frac{j}{2}, \delta_{l, R}$ has not yet been defined. But observe that $n_{v+l}$ belongs to $E_{j}(l, R)$, and thus it suffices to choose $\delta_{l, R}$ so that

$$
\frac{1}{L_{j}+(j+2) \times(j+1)} \geq\left(\prod_{i=1}^{j} \alpha_{i}\right) \delta_{l, R} .
$$

This finishes the construction.

Let $l \geq 1$ and $R>0$ be arbitrary; it now remains to prove that the set $E(l, R)$ has positive lower density. For $N \geq 1$, let

$$
E_{N}=\left\{n_{k} \leq N ; n_{k} \in E(l, R)\right\} .
$$

Let $j_{0}$ be an integer such that $l \leq j_{0}$ and $R \leq j_{0} / 2$, and let $N$ be any integer with $N \geq L_{j_{0}}$. There exists a $j>j_{0}$ with $L_{j-1} \leq N<L_{j}$. We evaluate the cardinal $\# E_{N}$ of $E_{N}$ using two different methods, according to the value of $N$.

Case 1: $L_{j-1} \leq N \leq L_{j-1}+(j+1) \times j$. Clearly $E_{j-1}(l, R) \subseteq E_{N}$, from which we deduce that

$$
\frac{\# E_{N}}{N} \geq \frac{\# E_{j-1}(l, R)}{L_{j-1}+(j+1) \times j} \geq\left(\prod_{i=1}^{j-1} \alpha_{i}\right) \delta_{l, R} \geq \frac{\delta_{l, R}}{2} .
$$

This explains why we required more than the simple assumption

$$
\frac{\# E_{j}(l, R)}{L_{j}} \geq\left(\prod_{i=1}^{j} \alpha_{j}\right) \delta_{l, R}
$$

in assertion (3) of the induction hypothesis: $L_{j-1}+(j+1) \times j$ is indeed the first integer where a shifted block of $B_{j-1}$ begins during the construction of $B_{j}$.

Case 2: $N>L_{j-1}+(j+1) \times j$. There exists an integer $p \geq 0$ such that

$$
(p+1)\left(L_{j-1}+j\right)+j^{2} \leq N<(p+2)\left(L_{j-1}+j\right)+j^{2} .
$$


But it follows from the construction that $E_{N}$ contains the set

$$
\begin{gathered}
E_{j-1}(l, R) \cup\left[\left(L_{j-1}+j\right)+j^{2}+E_{j-1}(l, R)\right] \\
\cup \cdots \cup\left[p\left(L_{j-1}+j\right)+j^{2}+E_{j-1}(l, R)\right] .
\end{gathered}
$$

This gives the following lower bound for $\# E_{N}$ :

$$
\# E_{N} \geq(p+1) \# E_{j-1}(l, R) \geq(p+1) \frac{\delta_{l, R}}{2} \times\left(L_{j-1}+(j+1) j\right) .
$$

On the other hand, we get an upper bound for $N$ by writing

$$
N \leq(p+2)\left(L_{j-1}+j(j+1)\right) .
$$

Hence

$$
\frac{\# E_{N}}{N} \geq \frac{\delta_{l, R}}{2} \frac{p+1}{p+2} \geq \frac{\delta_{l, R}}{4}
$$

from which it follows that $E(l, R)$ has positive lower density.

2.3. Examples. Theorem 2.1 and Lemma 2.2 give us our first examples of frequently hypercyclic operators.

Example 2.3. Let $B$ be the classical backward shift on $\ell^{p}$ with canonical basis $\left(e_{n}\right)_{n \geq 0}, 1 \leq p<+\infty$, defined by the relations $B e_{0}=0$ and $B e_{n}=e_{n-1}$ for $n \geq 1$. For any $\omega$ such that $|\omega|>1, \omega B$ is frequently hypercyclic on $\ell^{p}$. Indeed, take $\left(x_{l}\right)$ to be a dense sequence in $\ell^{p}$ of vectors whose support is finite, and define $S$ by $S e_{n}=\frac{1}{\omega} e_{n+1}$ for $n \geq 0$. Then $(\omega B) S=I,(\omega B)^{k} x_{l}=0$, provided $k$ is large enough, and $\left\|S^{k} x_{l}\right\| \leq \frac{1}{|\omega|^{k}}\left\|x_{l}\right\|$. Theorem 2.1 ensures that $\omega B$ is frequently hypercyclic. The frequent hypercyclicity of more general shifts will be investigated later on in Example 2.7.

Example 2.4. Let $\mathcal{H}(\mathbb{C})$ be the space of entire functions, endowed with the topology of uniform convergence on compact sets. MacLane proved in [40] the existence of an entire function having the property that the sequence of its derivatives is dense in $\mathcal{H}(\mathbb{C})$. In other words, the operator $D$ of differentiation is hypercyclic on $\mathcal{H}(\mathbb{C})$. But $D$ is in fact frequently hypercyclic.

Proof. Recall that $\mathcal{H}(\mathbb{C})$ is an $\mathcal{F}$-space with the usual metric

$$
\rho(f, g)=\sum_{n \geq 1} \frac{1}{2^{n}}\left(\frac{\sup _{|z| \leq n}|f(z)-g(z)|}{1+\sup _{|z| \leq n}|f(z)-g(z)|}\right)
$$

for $f, g \in \mathcal{H}(\mathbb{C})$. Let $D$ be the set of polynomials with coefficients in $\mathbb{Q}+i \mathbb{Q}$, and enumerate this dense set as $\left(P_{k}\right)_{k \geq 1}$. Of course $D^{n} P_{k}=0$ for $n$ large enough. The right-inverse $S$ is the operator of anti-derivation defined as

$$
S f(z)=\int_{(0, z)} f(\xi) d \xi
$$

for every $f \in \mathcal{H}(\mathbb{C})$, where $(0, z)$ is any path connecting 0 and $z$. Then $D S=$ $I$, and it is proved in [16] that for any $P_{k}$ in $D$, the series $\sum_{n \geq 1} \rho\left(S^{n} P_{k}, 0\right)$ is convergent.

Example 2.5. Let $T$ be Birkhoff's translation operator on $\mathcal{H}(\mathbb{C})$ (11]) defined by $T f(z)=f(z+1)$ for every $f \in \mathcal{H}(\mathbb{C})$. Then $T$ is frequently hypercyclic. 
Proof. For this example, it is convenient to give a direct proof involving Arakeljan's Theorem and our Lemma 2.2 again. We recall Arakeljan's Theorem below as Lemma 2.6

Lemma 2.6. Denote by $\bar{C}=\mathbb{C} \cup\{\infty\}$ the extended complex plane. Let $F \subset \mathbb{C}$ be a closed set such that $\overline{\mathbb{C}} \backslash F$ is connected and locally connected at $\infty$. Then for every function $g: F \rightarrow \mathbb{C}$, continuous on $F$ and holomorphic in its interior, there is an entire function $\varphi$ such that for every $z$ in $F$,

$$
|\varphi(z)-g(z)| \leq \exp \left(-|z|^{1 / 4}\right) \text {. }
$$

In fact, in the previous statement, $\exp \left(-t^{1 / 4}\right)$ can be replaced by any positive error function $\varepsilon(t)$ such that

$$
\int_{1}^{+\infty} t^{-3 / 2} \log \varepsilon(t) d t>-\infty
$$

So Arakeljan's Theorem allows us to approximate $g$ by $\varphi$ at infinity.

As in Theorem 2.1, consider the sequences $\left(n_{k}\right),\left(R_{k}\right)$ and $\left(m_{k}\right)$ given by Lemma 2.2. Enumerate the complex polynomials with coefficients in $\mathbb{Q}+i \mathbb{Q}$ as $\left(P_{k}\right)_{k \geq 1}$, and let $D_{k}$ be the closed disk of $\mathbb{C}$ with center $n_{k}$ and radius $R_{k} / 2$. These disks are disjoint, which makes it possible to define a function $g$ on the closed set $F=$ $\bigcup_{k \geq 1} D_{k}$ by the formula

$$
g(z)=P_{m_{k}}\left(z-n_{k}\right) \text { if } z \text { belongs to } D_{k} .
$$

Since $\overline{\mathbb{C}} \backslash F$ is connected and locally connected at $\infty$, Arakeljan's Theorem can be applied to obtain an entire function $\varphi$ such that

$$
|\varphi(z)-g(z)| \leq \exp \left(-|z|^{1 / 4}\right)
$$

for every $z \in F$. We claim that $\varphi$ is a frequently hypercyclic vector for $T$. Indeed, let $K$ be any compact subset of $\mathbb{C}, f$ any entire function, and $\varepsilon>0$. One can find $l \geq 1$ and $R>0$ such that $\left\|P_{l}-f\right\|_{C(K)}<\varepsilon / 2$ and $K \subset D(0, R / 2)$. Take $n=n_{k}$ in $E(l, R)$ and $z$ in $K$. Then

$$
\begin{aligned}
\left|T^{n} \varphi(z)-f(z)\right| & \leq\left|\varphi\left(z+n_{k}\right)-f(z)\right| \\
& \leq\left|\varphi\left(z+n_{k}\right)-g\left(z+n_{k}\right)\right|+\left|P_{l}(z)-f(z)\right| \\
& \leq \frac{\varepsilon}{2}+\exp \left(-\left|n_{k}-R\right|^{1 / 4}\right) .
\end{aligned}
$$

In other words, if $n_{k}$ belonging to $E(l, R)$ is large enough, then $\left\|T^{n_{k}} \varphi-f\right\|_{C(K)}$ $<\varepsilon$, which proves our claim.

Example 2.7. Let $\left(e_{n}\right)_{n \geq 0}$ be the canonical basis of one of the spaces $\ell_{p}, 1 \leq p<$ $+\infty$, and $\left(\omega_{n}\right)_{n \geq 1}$ a bounded sequence of positive numbers, bounded away from 0 . Let $B$ be the backward shift on $\ell_{p}$ with weights $\left(\omega_{n}\right): B e_{0}=0$ and $B e_{n}=\omega_{n} e_{n-1}$ for $n \geq 1$. If the series

$$
\sum_{n \geq 1} \frac{1}{\left(\omega_{1} \ldots \omega_{n}\right)^{p}}
$$

is convergent, then $B$ is frequently hypercyclic. 
Proof. Suppose that the series above is convergent. Theorem 2.1 cannot be applied directly (this would be the case under the stronger assumption that the series $\sum_{n \geq 1} \frac{1}{\omega_{1} \ldots \omega_{n}}$ converges), but the same method of proof applies. Let $\left(n_{k}\right),\left(m_{k}\right)$ and $\left(R_{k}\right)$ be the sequences obtained in Lemma 2.2. Remark that $m_{k} \leq 2 R_{k}$ (this is clear from the proof of Lemma 2.2), hence $n_{k+1}-n_{k} \geq m_{k} / 2$. Let $D$ be the set of finitely supported vectors of $\ell_{p}$ with coordinates in $\mathbb{Q}+i \mathbb{Q}$, and write $D$ as $\left(x_{l}\right)_{l \geq 1}$, assuming moreover that the support of $x_{l}$, denoted by $\operatorname{supp}\left(x_{l}\right)$, is a subset of $\left[0, l / 2\left[\right.\right.$. Let $S$ be the forward shift defined by $S e_{n}=\frac{1}{\omega_{n+1}} e_{n+1}$, and $\gamma, C$ two positive constants with $\gamma \leq\left|\omega_{n}\right| \leq C$ for any $n$. Then $\operatorname{supp}\left(y_{k}\right) \subseteq\left[0, m_{k} / 2[\right.$, where the $y_{k}$ 's are the vectors that appear in the proof of Theorem 2.1. This implies that the supports of the vectors $S^{n_{k}} y_{k}$ are successive and disjoint $\left(\max \operatorname{supp}\left(S^{n_{k}} y_{k}\right)<\right.$ $\left.\min \operatorname{supp}\left(S^{n_{k+1}} y_{k+1}\right)\right)$. Hence $\|x\|^{p}=\sum\left\|S^{n_{k}} y_{k}\right\|^{p}$. Now

$$
\left\|S^{n_{k}} x_{l}\right\|^{p} \leq \frac{1}{\left(\omega_{1} \ldots \omega_{n_{k}}\right)^{p}}\left(\frac{C}{\gamma}\right)^{l}\left\|x_{l}\right\|^{p}
$$

and the series $\sum_{k>1}\left\|S^{n_{k}} x_{l}\right\|^{p}$ is convergent. If $\left(N_{l}\right)_{l \geq 1}$ is a strictly increasing sequence such that for every $l$ and every $j \leq l$,

$$
\sum_{n_{k} \geq N_{l}}\left\|S^{n_{k}} x_{l}\right\|^{p} \leq \frac{1}{2^{j+l}}
$$

then

$$
\sum_{k \geq 1}\left\|S^{n_{k}} y_{k}\right\|^{p}=\sum_{l \geq 1} \sum_{\substack{m_{k}=l \\ R_{k} \geq N_{l}}}\left\|S^{n_{k}} x_{l}\right\|^{p} \leq \sum_{l \geq 1} \sum_{n_{k} \geq N_{l}}\left\|S^{n_{k}} x_{l}\right\|^{p} \leq \frac{1}{4^{l}},
$$

and $x$ is well defined. Since the vectors $S^{n-n_{k}} y_{k}$, or $B^{n-n_{k}} y_{k}$, that appear in the sequel of the proof have successive supports again, the rest of the proof follows along the same lines, and $x$ is easily seen to be a frequently hypercyclic vector for $B$.

On the other hand, it is easy to exhibit hypercyclic operators which are not frequently hypercyclic.

Example 2.8. With the same notation as above, let $B$ be the backward shift on $\ell_{p}$ with weights $\left(\omega_{n}\right)$. If for every sequence $\left(n_{k}\right)_{k \geq 1}$ with positive lower density the series

$$
\sum_{k \geq 1} \frac{1}{\left(\omega_{1} \ldots \omega_{n_{k}}\right)^{p}}
$$

is divergent, then $B$ is not frequently hypercyclic.

Proof. Suppose that $x$ is a frequently hypercyclic vector for $B$ and let $\left\{n_{k}\right\}$ be the set of integers such that $\left\|B^{n_{k}} x-e_{0}\right\|<\frac{1}{2}$. Then

$$
\left|x_{n_{k}}\right|^{p} \geq \frac{1}{2\left(\omega_{1} \ldots \omega_{n_{k}}\right)^{p}} .
$$

But the series $\sum\left|x_{n_{k}}\right|^{p}$ is convergent, and hence $\left\{n_{k}\right\}$ cannot have positive lower density, a contradiction.

For instance:

Example 2.9. The backward shift on $\ell_{2}$ with weight $\omega_{n}=\sqrt{1+\frac{1}{n}}, n \geq 1$, is hypercyclic but not frequently hypercyclic. 
Proof. We have $\left(\omega_{1} \ldots \omega_{n}\right)^{2}=n+1$. If $E$ has positive lower density, there exists a positive $\delta$ such that, for any $N_{0}$ in $\mathbb{N}$, there exists $N_{1} \geq N_{0}$ with $\#\left\{N_{0} \leq n+1 \leq N_{1}\right.$; $n \in E\} \geq \delta N_{1}$. This implies

$$
\sum_{\substack{N_{0} \leq n \leq N_{1} \\ n \in E}} \frac{1}{n+1} \geq \delta N_{1} \times \frac{1}{N_{1}}=\delta .
$$

The series

$$
\sum_{n \in E} \frac{1}{n+1}
$$

diverges: $B$ is not frequently hypercyclic.

Remark that $B$ can be seen as the backward shift on the Bergman space $A^{2}$. The fact that $B$ is hypercyclic when it operates on $A^{2}$ was shown in [26]; see also 27. Remark also that this shift is frequently hypercyclic on $\ell_{p}$ if and only if $p>2$. In particular, frequently hypercyclic shifts are not the same on each $\ell_{p}$, a situation which does not appear if we only consider the hypercyclicity phenomenon: it is known that the backward shift on $\ell_{p}$ with weights $\left(\omega_{n}\right)$ is hypercyclic if and only if there exists a sequence $\left(n_{k}\right)$ such that $\omega_{1} \ldots \omega_{n_{k}}$ tends to infinity (47]). It would be interesting to have a characterization of the frequently hypercyclic backward shifts on $\ell_{p}$. In view of Examples 2.7 and 2.8, the following conjecture seems plausible.

Conjecture 2.10. The backward shift $B$ with weight $\left(\omega_{n}\right)_{n \geq 1}$ is frequently hypercyclic on $\ell_{p}$ if and only if there exists a sequence $\left(n_{k}\right)_{k \geq 1}$ with positive lower density such that the series

$$
\sum_{k \geq 1} \frac{1}{\left(\omega_{1} \ldots \omega_{n_{k}}\right)^{p}}
$$

is convergent.

\section{The ROLE OF The Unimodular POINT SPECTRUM}

3.1. Main result. In this section, we restrict ourselves to the case when the underlying space is a complex Hilbert space $H$ of infinite dimension. Our aim is to show that operators with sufficiently many eigenvectors associated to unimodular eigenvalues are frequently hypercyclic. Let us first make this assumption on eigenvectors precise.

Definition 3.1. Let $T$ be a bounded operator on $H$. We say that $T$ has a perfectly spanning set of eigenvectors associated to unimodular eigenvalues if there exists a continuous probability measure $\sigma$ on the unit circle $\mathbb{T}$ such that for every $\sigma$ measurable subset $A$ of $\mathbb{T}$ with $\sigma(A)=1$, the eigenspaces $\operatorname{ker}(T-\lambda I), \lambda \in A$, span a dense subspace of $H$. Here $\sigma$ is said to be continuous if $\sigma(\{\lambda\})=0$ for every $\lambda \in \mathbb{T}$.

Our aim will be to prove the following theorem.

Theorem 3.2. If $T$ has a perfectly spanning set of eigenvectors associated to unimodular eigenvalues, then $T$ is frequently hypercyclic. In particular $T$ is hypercyclic. It is even weakly topologically mixing: if $U$ and $V$ are two nonempty open subsets of $H$, there exists a sequence $\left(n_{k}\right)$ of integers of density 1 such that $T^{n_{k}}(U) \cap V$ is nonempty for every $k$. 
Several remarks are in order before we launch ourselves in the proof of Theorem 3.2 first of all, Theorem 3.2 implies that any operator with a perfectly spanning set of eigenvectors associated to unimodular eigenvalues is hypercyclic. This is not obvious, and a direct proof which is also valid in the Banach space setting is given in [5]; see also 4] for a simplified proof in a special case. This hypercyclicity result can be seen as a counterpart to a criterion of Godefroy and Shapiro (27]), which runs as follows.

Godefroy-Shapiro Criterion. Suppose that the eigenspaces $\operatorname{ker}(T-\lambda I),|\lambda|>1$, span a dense subspace of $H$ as well as the eigenspaces $\operatorname{ker}(T-\lambda I),|\lambda|<1$. Then $T$ is hypercyclic.

Here we consider the eigenspaces associated to unimodular eigenvalues, but a simple density assumption is not sufficient to obtain hypercyclicity: any diagonal operator on a Hilbert space with unimodular diagonal coefficients has a spanning set of eigenvectors associated to unimodular eigenvalues, but is very far from being hypercyclic. Theorem 3.2 is rather easy to apply, and there is a simple argument which gives us a whole bunch of operators with a perfectly spanning set of eigenvectors associated to unimodular eigenvalues.

Example 3.3. Let $B$ be the backward shift on $\ell_{2}$ with canonical basis $\left(e_{n}\right)_{n \geq 0}$. Then for any $\omega$ such that $|\omega|>1, \omega B$ has a perfectly spanning set of eigenvectors associated to unimodular eigenvalues.

Proof. It is easy to see that $\lambda$ is an eigenvalue of $\omega B$ if and only if $|\lambda|<|\omega|$, and that in this case $\operatorname{ker}(\omega B-\lambda I)$ is the 1-dimensional space spanned by the vector

$$
x_{\lambda}=\sum_{n=0}^{+\infty}\left(\frac{\lambda}{\omega}\right)^{n} e_{n} .
$$

Let $\sigma$ be the normalized length measure on the unit circle $\mathbb{T}: d \sigma=\frac{1}{2 \pi} d \theta$, and suppose that $A$ is a subset of $\mathbb{T}$ of full measure. Let $x$ be such that $\left\langle x, x_{\lambda}\right\rangle=0$ for every $\lambda \in A$. Then the analytic function $\Phi$ defined on $D(0,|\omega|)$ by

$$
\Phi(\lambda)=\sum_{n=0}^{+\infty}\left\langle x, e_{n}\right\rangle\left(\frac{\lambda}{\omega}\right)^{n}
$$

vanishes on $A$. Since $A$ is an uncountable subset of $\mathbb{T}, A$ has an accumulation point in $D(0,|\omega|)$, hence $\Phi$ is identically zero, $\left\langle x, e_{n}\right\rangle=0$ for every $n \geq 0$ and $x=0$. The kernels $\operatorname{ker}(\omega B-\lambda I), \lambda \in A$, span a dense subspace of $\ell_{2}$.

Example 3.4. Let $T$ be any upper-triangular operator on $\ell_{2}$ with respect to an orthonormal basis $\left(e_{n}\right)_{n>0}$, and $B$ the backward shift with respect to this same basis. For every $\omega$ such that $|\omega|>\max (1,\|T\|), T+\omega B$ has a perfectly spanning set of eigenvectors associated to unimodular eigenvalues.

Proof. This is basically the same argument as above; see [29] for details.

Example 3.5. Let $\Omega$ be a connected open subset of $\mathbb{C}$, and $H$ a nontrivial Hilbert space of analytic functions on $\Omega$ such that the point evaluations $f \longmapsto f(z)$ are bounded for every $z \in \Omega$. Let $\phi$ be a multiplier, i.e. a function such that for every $f \in H, \phi f$ is also in $H$. This multiplier defines a linear multiplication operator $M_{\phi}$ by the formula

$$
M_{\phi}(f)=\phi f
$$


for $f \in H$. The hypercyclicity of adjoints of multipliers was studied by Godefroy and Shapiro in [27, where it was shown that $M_{\phi}^{*}$ is hypercyclic whenever $\phi$ is a nonconstant multiplier such that $\phi(\Omega)$ intersects the unit circle. In fact every such $M_{\phi}^{*}$ has a perfectly spanning set of eigenvectors associated to unimodular eigenvalues.

Proof. Let $\gamma$ be a nonempty open subarc contained in $\overline{\phi(\Omega)} \cap \mathbb{T}$, where $\overline{\phi(\Omega)}$ denotes the set of complex conjugates of elements of $\phi(\Omega)$. Let $\sigma$ be the normalization of the length measure restricted to $\gamma$ :

$$
d \sigma=\frac{1}{l(\gamma)} \mathbb{1}_{\gamma} d \theta
$$

For every $z \in \Omega$, let $k_{z}$ be the reproducing kernel defined by the relation $f(z)=$ $\left\langle f, k_{z}\right\rangle$ for every $f \in H$. Then $M_{\phi}^{*} k_{z}=\overline{\phi(z)} k_{z}$. Thus every element of $\gamma$ is an eigenvalue of $M_{\phi}^{*}$. Suppose now that $A$ is a subset of $\mathbb{T}$ which satisfies $\sigma(A)=1$, and that $f$ is a function in $H$ such that $\left\langle f, k_{z}\right\rangle=0$ whenever $\overline{\phi(z)}$ belongs to $A$. Then $f(z)=0$ whenever $z$ belongs to $\phi^{-1}(\bar{A})$ (here $\bar{A}$ denotes the conjugate set of $A)$. Now $\phi^{-1}(\bar{A})$ is an uncountable subset of $\Omega$, hence $\phi^{-1}(\bar{A})$ has an accumulation point in $\Omega$ and $f$ vanishes identically on $\Omega$. This proves our claim.

Example 3.6. Let $\phi$ be an automorphism of the unit disk $\mathbb{D}$, and $C_{\phi}$ the composition operator defined on $H^{2}(\mathbb{D})$ by the formula $C_{\phi}(f)=f \circ \phi$. This operator is bounded on $H^{2}(\mathbb{D})$, and it is hypercyclic if and only if $\phi$ has no fixed point in the unit disk $\mathbb{D}([15)$. The automorphism $\phi$ is said to be parabolic when it has exactly one (attractive) fixed point in $\mathbb{T}$, and hyperbolic when it has exactly two fixed points in $\mathbb{T}$ (one of which is exactly attractive). We will assume that 1 is the attractive fixed point of the automorphism $\phi$. Then, it is easier to describe such an automorphism using the map $\psi=\theta \circ \phi \circ \theta^{-1}$ instead of $\phi$ on the right half-plane $\mathbb{C}_{+}$, where $\theta$ is the Cayley map from $\mathbb{D}$ onto $\mathbb{C}_{+}$defined by

$$
\theta(z)=\frac{1+z}{1-z}
$$

When $\phi$ is a parabolic automorphism, $\psi$ is a translation operator on $\mathbb{C}_{+}$:

$$
\psi(z)=T_{a}(z)=z+i a,
$$

with $a \in \mathbb{R}$ and $a \neq 0$ (a parabolic automorphism is conjugate to a translation), and when $\phi$ is a hyperbolic automorphism, then

$$
\psi(z)=h_{\lambda, b}(z)=\lambda(z-i b)+i b,
$$

with $\lambda>1$ and $b \in \mathbb{R}$ (a hyperbolic automorphism is conjugate to a dilation). Parabolic and hyperbolic composition operators have a perfectly spanning set of eigenvectors associated to unimodular eigenvalues.

Proof. Suppose that $\phi$ is a parabolic automorphism conjugated to $T_{a}$, and for $t \geq 0$, let

$$
e_{t}(z)=\exp \left(-t \frac{1+z}{1-z}\right)
$$

Then $e_{t}$ is an eigenfunction for $C_{\phi}$ associated to the eigenvalue $e^{-i t a}$. It is well known that the functions $e_{t}, t \geq 0$, span a dense subspace of $H^{2}(\mathbb{D}$ ) (for a discussion about the origin of this result, see [24, p. 53]; a simple proof due to D. Sarason can be found in [25]). Take $\sigma$ to be the normalized length measure on $\mathbb{T}$, and let 
$A$ be a measurable subset of $\mathbb{T}$ with $\sigma(A)=1$. If $\left\langle f, e_{t}\right\rangle=0$ for every $t$ such that $e^{-i t a}$ is in $A$, then $\left\langle f, e_{t}\right\rangle=0$ for every $t$ in a dense subset of $\mathbb{R}^{+}$. Since $e_{t}$ depends continuously on $t,\left\langle f, e_{t}\right\rangle$ vanishes for every $t$, and hence $f=0$.

The following proof for a hyperbolic automorphism was kindly pointed out to us by the referee. It simplifies our earlier proof which was based on results of Nordgren, Rosenthal and Wintrobe ([44]). So, suppose that $\phi$ is a hyperbolic automorphism whose Denjoy-Wolff point is 1 , and set $\lambda=\frac{1}{\phi^{\prime}(1)}$. Observe that for each real number $r$,

$$
f_{r}(z)=\exp \left(\frac{i r}{\log (\lambda)} \log \left(\frac{1+z}{1-z}\right)\right)
$$

is an eigenvector for $C_{\phi}$ with corresponding eigenvalue $e^{i r}$. Each function $f_{r}$ is analytic and bounded on $\mathbb{D}$, hence belongs to $H^{2}(\mathbb{D})$. With the same notation as above, suppose that $A$ is a measurable subset of $\mathbb{T}$ with $\sigma(A)=1$, and that for some $g \in H^{2}(\mathbb{D}),\left\langle f_{r}, g\right\rangle=0$ for every $r$ such that $e^{i r}$ belongs to $A$. Since the function $r \mapsto\left\langle f_{r}, g\right\rangle$ is analytic in a neighborhood of the origin, this implies that its Taylor coefficients at 0 must vanish: for every $n \geq 0$,

$$
\left\langle\left(\log \left(\frac{1+z}{1-z}\right)\right)^{n}, g\right\rangle=0 .
$$

These powers are dense in $H^{2}([15]$, Theorem 3.8), so it follows that $g=0$.

Thus Theorem 3.2 yields:

Corollary 3.7. All the operators considered in the previous examples (multiples of the backward shift, perturbations of upper-triangular operators by big multiples of a backward shift, adjoints of multipliers on spaces of holomorphic functions, parabolic and hyperbolic composition operators) are frequently hypercyclic.

It is interesting to note that all the operators exhibited in [29] and [30] have in fact a perfectly spanning set of unimodular eigenvectors. This implies the following.

Corollary 3.8. Every bounded operator on a separable Hilbert space can be written as the sum of two frequently hypercyclic operators. Every bounded operator on one of the spaces $\ell_{p}, 1 \leq p<+\infty$, can be written as the sum of six frequently hypercyclic operators.

The rest of Section 3 is devoted to the proof of Theorem 3.2. Our strategy is to use a relation between topological dynamics (here, frequent hypercyclicity) and ergodic theory.

3.2. Ergodic theory and frequent hypercyclicity. Let us first recall some basic definitions from ergodic theory. For more information on this topic, see for instance [50].

Definition 3.9. The measure-preserving transformation $T:(H, \mathcal{B}, m) \rightarrow\left(H, \mathcal{B}^{\prime}, m\right)$ is said to be ergodic when one of the following equivalent assertions is satisfied:

(1) for every element $A, B$ of $\mathcal{B}$ such that $m(A)>0$ and $m(B)>0$, there exists an integer $n$ such that $m\left(T^{-n}(A) \cap B\right)>0$,

(2) if $B$ is an element of $\mathcal{B}$ which satisfies $T^{-1}(B)=B$, then $m(B)=0$ or $m(B)=1$, 
(3) for every $A, B \in \mathcal{B}$,

$$
\lim _{N \rightarrow \infty} \frac{1}{N} \sum_{k=0}^{N-1} m\left(T^{-k}(A) \cap B\right)=m(A) m(B),
$$

(4) for every $f, g \in L^{2}(H, \mathcal{B}, m)$,

$$
\lim _{N \rightarrow \infty} \frac{1}{N} \sum_{k=0}^{N-1}\left\langle U_{T}^{k} f, g\right\rangle=\langle f, 1\rangle \overline{\langle g, 1\rangle .}
$$

The fact that assertions (3) and (4) are equivalent to assertions (1) and (2) uses Birkoff's ergodic theorem. Changing the mode of convergence in assertions (3) and (4) above gives the notions of weak-mixing and strong-mixing transformations, which will be of interest to us in the sequel.

Definition 3.10. The measure-preserving transformation $T:(H, \mathcal{B}, m) \rightarrow\left(H, \mathcal{B}^{\prime}, m\right)$ is said to be weak-mixing when one of the following equivalent assertions is satisfied:

(1) for every $A, B \in \mathcal{B}$,

$$
\lim _{N \rightarrow \infty} \frac{1}{N} \sum_{k=0}^{N-1}\left|m\left(T^{-k}(A) \cap B\right)-m(A) m(B)\right|=0,
$$

(2) for every $f, g \in L^{2}(H, \mathcal{B}, m)$,

$$
\lim _{N \rightarrow \infty} \frac{1}{N} \sum_{k=0}^{N-1}\left|\left\langle U_{T}^{k} f, g\right\rangle-\langle f, 1\rangle \overline{\langle g, 1\rangle}\right|=0 .
$$

Definition 3.11. The measure-preserving transformation $T:(H, \mathcal{B}, m) \rightarrow\left(H, \mathcal{B}^{\prime}, m\right)$ is said to be strong-mixing when one of the following equivalent assertions is satisfied:

(1) for every $A, B \in \mathcal{B}$,

$$
\lim _{n \rightarrow \infty} m\left(T^{-n}(A) \cap B\right)=m(A) m(B),
$$

(2) for every $f, g \in L^{2}(H, \mathcal{B}, m)$,

$$
\lim _{n \rightarrow \infty}\left\langle U_{T}^{n} f, g\right\rangle=\langle f, 1\rangle \overline{\langle g, 1\rangle} .
$$

Of course a strong-mixing transformation is weak-mixing, and a weak-mixing transformation is ergodic. Moreover, $T$ is weak-mixing if and only if $T \times T$ is ergodic on $(X \times X, \mathcal{B} \otimes \mathcal{B}, m \otimes m)$ (see for instance [50, p. 46]).

The next proposition explains why ergodic theory is of interest to us in this setting.

Proposition 3.12. Let $T$ be a bounded operator on $H$. Suppose that there exists a measure $m$ on $(H, \mathcal{B}, m)$ whose support is $H$ and such that $T:(H, \mathcal{B}, m) \longrightarrow$ $(H, \mathcal{B}, m)$ is a measure-preserving ergodic transformation. Then $T$ is frequently hypercyclic, and the set $F H C(T)$ has m-measure 1.

Proof. Let $\left(U_{p}\right)_{p \geq 1}$ be a countable basis of nonempty open subsets of $X$. Birkhoff's ergodic theorem ensures that for $m$-almost every $x$ in $X$,

$$
\frac{1}{N} \#\left\{k \leq N ; T^{k} x \in U_{p}\right\} \longrightarrow m\left(U_{p}\right)
$$


as $N$ tends to infinity. Since $m\left(U_{p}\right)$ is positive for every $p$, it follows that $m$-almost every $x$ in $X$ is frequently hypercyclic for $T$.

So our interest lies in constructing (if such a thing is possible) an invariant measure on $H$ for a bounded operator $T$ with respect to which $T$ is ergodic, where we additionally require that the support of the measure be the whole space $H$. Gaussian measures are especially well suited to this purpose. Questions of this kind were first investigated by Flytzanis in his paper 21. (see also 22]), the crucial tool being the intertwining equation $T K=K V$, which will be of constant use in the sequel. Proposition 3.18 below is stated in [21, as well as a first version of Theorem 3.22 under somewhat different assumptions on the eigenvectors, and when $T$ verifies some supplementary conditions ( $T$ has to be injective with simple eigenvalues). Some complements are given in [22] to the effect that these additional assumptions can be removed. Since several points in the argument in 21] are rather difficult to follow, we give below a complete self-contained exposition of a proof of Theorem 3.22. This new proof also has the advantage that it can be extended to the Banach space setting under suitable assumptions on the geometry of the space; see [6]. As for Proposition 3.18, a proof is sketched in the announcement [20, and Section 3.3 below, which is mostly expository, expands on this.

3.3. Gaussian measures on Hilbert spaces. We begin by recalling some definitions and facts about Gaussian measures on complex Hilbert spaces. All the definitions and facts on Gaussian measures stated below can be found in one of the references [39, 12] or 35].

Definition 3.13. Let $(\Omega, \mathcal{F}, P)$ be a probability space and $f:(\Omega, \mathcal{F}, P) \longrightarrow \mathbb{C}$ a complex-valued measurable function. Then $f$ is said to have complex symmetric Gaussian distribution if the real and imaginary parts $\Re e f$ and $\Im m f$ of $f$ have independent centered Gaussian distribution with the same variance.

This is equivalent to saying that $\Re e f$ and $\Im m f$ are jointly normal and that $f$ and $\lambda f$ have the same distribution for any $\lambda$ of modulus 1 ([35, p. 13]).

Definition 3.14. Let $H$ be a complex Hilbert space. A Gaussian measure on $H$ is a probability measure $m$ on $H$ such that for every $x \in H$, the function $f_{x}: y \longmapsto\langle y, x\rangle$ has symmetric complex Gaussian distribution.

In particular with this terminology, such a measure is centered:

$$
\int_{H}\langle y, x\rangle d m(y)=\int_{H} f_{x}(y) d m(y)=\int_{H} y d\left(f_{x}(m)\right)(y)=0 .
$$

Moreover the quantity

$$
\int_{H}\|z\|^{2} d m(z)
$$

is always finite. A Gaussian measure is completely determined by its covariance operator $S$ defined on $H$ by the relation

$$
\langle S x, y\rangle=\int_{H}\langle x, z\rangle \overline{\langle y, z\rangle} d m(z) \quad \text { for every } x, y \in H .
$$

Since $m$ has a moment of order two, $S$ is a bounded self-adjoint positive operator on $H$. Moreover, $S$ is of trace class, and the support of the measure $m$ is the normclosure of the range of $S$. Thus the support of $m$ is the whole space if and only if $S$ is injective. In this case we say that the measure $m$ is nondegenerate. Conversely, 
if $S$ is a bounded self-adjoint positive operator of trace class, there exists a unique Gaussian measure on $H$ whose covariance operator is $S$. This shows that finding an invariant Gaussian measure for an operator boils down to solving a certain operator equation.

Proposition 3.15. Let $T$ be a bounded operator on $H$. The following assertions are equivalent:

(1) $T$ admits an invariant nondegenerate Gaussian measure,

(2) there exists a bounded self-adjoint positive operator $S$ of trace class which is injective and satisfies $T S T^{*}=S$,

(3) there exist a Hilbert space $G$, a compact operator $K: G \rightarrow H$ which is Hilbert-Schmidt with dense range and a co-isometry $V: G \rightarrow G$ such that $T K=K V$ (and hence $\left.K^{*} T^{*}=V^{*} K^{*}\right)$.

The equation above was introduced by Flytzanis in 21, and will be referred to as equation

$$
T K=K V .
$$

Proof. If $m$ is a Gaussian measure on $H, T(m)$ is also a Gaussian measure on $H$. Let $S^{\prime}$ be its covariance operator. Then

$$
\left\langle S^{\prime} x, y\right\rangle=\int_{H}\langle x, z\rangle \overline{\langle y, z\rangle} d(T(m))(z)=\int_{H}\left\langle T^{*} x, z\right\rangle \overline{\left\langle T^{*} y, z\right\rangle} d m(z)=\left\langle T S T^{*} x, y\right\rangle,
$$

hence $S^{\prime}=T S T^{*}$. The equivalence of assertions (1) and (2) then follows directly from the fact that a centered Gaussian measure is completely determined by its covariance operator.

$(3) \Rightarrow(2)$ is easy: set $S=K K^{*}$. It is a bounded self-adjoint positive operator, it is injective since $K$ has dense range, and since $K^{*}$ is Hilbert-Schmidt, $S$ is of trace class. Moreover $T S T^{*}=T K K^{*} T^{*}=K V V^{*} K^{*}=S$, which proves (2).

$(2) \Rightarrow(3)$ : consider the polar decomposition of $(T \sqrt{S})^{*}$ : there exist a selfadjoint operator $P$ and an isometry $W$ such that $(T \sqrt{S})^{*}=W P$. Set $V=W^{*}$ : $P$ being the unique positive square root of $(T \sqrt{S})(T \sqrt{S})^{*}=S, P=\sqrt{S}$ and $\sqrt{S} T^{*}=V^{*} \sqrt{S}$, i.e. $T \sqrt{S}=\sqrt{S} V$. Setting $K=\sqrt{S}$ yields equation $(F)$. It is clear that $K$ is Hilbert-Schmidt and injective, and since $K$ is self-adjoint, it also has dense range.

Unimodular eigenvectors are the main tool we use to construct Gaussian invariant measures. We need one more definition here.

Definition 3.16. Let $T$ be a bounded operator on $H$, and let $\sigma$ be a probability measure on $\mathbb{T}$. Then $T$ is said to have a $\sigma$-spanning set of eigenvectors associated to unimodular eigenvalues if for every Borel subset $A$ of $\mathbb{T}$ with $\sigma(A)=1$, the eigenspaces $\operatorname{ker}(T-\lambda I)$ for $\lambda \in A$ span a dense subspace of $H$.

Thus $T$ has a perfectly spanning set of eigenvectors associated to unimodular eigenvalues if and only of it has a $\sigma$-spanning set of eigenvectors associated to unimodular eigenvalues for some continuous measure $\sigma$. The eigenspaces can be described via countably many Borel functions.

Lemma 3.17. Let $T$ be a bounded operator on $H$. There exists a sequence of Borel measurable and bounded functions $E_{i}: \mathbb{T} \rightarrow B_{H}, i \geq 1$, such that for every $\lambda \in \mathbb{T}$, the span of the sequence of vectors $\left(E_{i}(\lambda)\right)_{i \geq 1}$ is dense in $\operatorname{ker}(T-\lambda I)$. 
Here $B_{H}$ denotes the closed unit ball of $H$. A stronger result was obtained by Dixmier and Foias in [17, where it is shown that the functions $\left(E_{i}\right)_{i \geq 1}$ can be chosen so that

(1) if $\operatorname{ker}(T-\lambda I)$ has finite dimension $d_{\lambda}, E_{i}(\lambda)=0$ for $i>d_{\lambda}$, and

(2) for every $\lambda \in \mathbb{T}$, the sequence of vectors $\left(E_{i}(\lambda)\right)_{i \geq 1}$ is an orthonormal basis of the eigenspace $\operatorname{ker}(T-\lambda I)$.

Results of this kind were also obtained by Nikolskaya in 42 and [43. Here we give a simple direct proof of Lemma 3.17

Proof. Let $\left(x_{i}\right)$ be a dense sequence of vectors of the unit sphere of $H$, and for $\lambda \in \mathbb{T}$, denote by $P_{\lambda}$ the orthogonal projection onto $\operatorname{ker}(T-\lambda I)$. Let $E_{i}$ be defined on $\mathbb{T}$ by $E_{i}(\lambda)=P_{\lambda} x_{i}$. Then $E_{i}$ is a Borel map on $\mathbb{T}$ (this follows directly from the fact that $\lambda \mapsto\left\|P_{\lambda} x\right\|$ is upper semi-continuous for every $x \in H$; see for instance [17]) and the closed linear span of the vectors $E_{i}(\lambda)$ is exactly the eigenspace $\operatorname{ker}(T-\lambda I)$. Clearly each $E_{i}(\lambda)$ is in $\operatorname{ker}(T-\lambda I)$, and if $x$ is orthogonal to $P_{\lambda} x_{i}$ for every $i, x$ is orthogonal to the range of $P_{\lambda}$.

Proposition 3.18. Suppose that $T$ has a $\sigma$-spanning set of eigenvectors associated to unimodular eigenvalues. Then $T$ satisfies equation $(F)$. In particular $T$ has a nondegenerate invariant Gaussian measure.

Proof. Let $\left(E_{i}\right)_{i \geq 1}$ be the sequence of eigenvector fields given by Lemma 3.17, Let $V$ be defined on the space $\bigoplus_{i \geq 1} L^{2}(\mathbb{T}, \sigma)$ of sequences $\left(f_{i}\right)_{i \geq 1}$ of functions of $L^{2}(\mathbb{T}, \sigma)$ such that $\sum_{i \geq 1}\left\|f_{i}\right\|^{2}<+\infty$ by

$$
V\left(\bigoplus_{i \geq 1} f_{i}\right)(\lambda)=\bigoplus_{i \geq 1} \lambda f_{i}(\lambda)
$$

In other words, $V$ acts as the multiplication operator by $\lambda$ on each component $L^{2}(\mathbb{T}, \sigma)$. It is clearly a unitary operator. Now let $K: \bigoplus_{i \geq 1} L^{2}(\mathbb{T}, \sigma) \rightarrow H$ be defined by

$$
K\left(\bigoplus_{i \geq 1} f_{i}\right)=\sum_{i=1}^{+\infty} \frac{1}{2^{i}} \int_{\mathbb{T}} f_{i}(\lambda) E_{i}(\lambda) d \sigma(\lambda) .
$$

Then $K$ is a well-defined Hilbert-Schmidt operator: each $K_{i}: L^{2}(\mathbb{T}, \sigma) \rightarrow H$ which maps $f_{i}$ onto $\int_{\mathbb{T}} f_{i}(\lambda) E_{i}(\lambda) d \sigma(\lambda)$ is a kernel operator with a square-summable kernel. Let us now show that $K$ has dense range, and suppose to this aim that $x \in H$ satisfies $\left\langle x, K\left(\bigoplus_{i \geq 1} f_{i}\right)\right\rangle=0$ for every square-summable sequence $\left(f_{i}\right)_{i \geq 1}$. Taking all the elements in this sequence to be 0 except one which is arbitrary, this yields that for every $i \geq 1$ and every $f \in L^{2}(\mathbb{T}, \sigma)$,

$$
\left\langle x, \int_{\mathbb{T}} f(\lambda) E_{i}(\lambda) d \sigma(\lambda)\right\rangle=0 .
$$

This in turn implies that $\left\langle x, E_{i}(\lambda)\right\rangle=0 \sigma$-almost surely, which means that $x$ is orthogonal to $\operatorname{ker}(T-\lambda I)$ except for $\lambda$ in a set of $\sigma$-measure 0 . The assumption that $T$ has a $\sigma$-spanning set of eigenvectors implies that $x=0$, and $K$ has dense range. It remains to check that equation $(F)$ is satisfied with this choice of the 
pair $(K, V)$. Using the fact that $E_{i}(\lambda)$ belogs to $\operatorname{ker}(T-\lambda I)$, we get that for every $\left(f_{i}\right)_{i \geq 1} \in \bigoplus_{i \geq 1} L^{2}(\mathbb{T}, \sigma)$,

$$
\begin{aligned}
T K\left(\bigoplus_{i \geq 1} f_{i}\right) & =\sum_{i=1}^{+\infty} \frac{1}{2^{i}} \int_{\mathbb{T}} f_{i}(\lambda) T E_{i}(\lambda) d \sigma(\lambda)=\sum_{i=1}^{+\infty} \frac{1}{2^{i}} \int_{\mathbb{T}} f_{i}(\lambda) \lambda E_{i}(\lambda) d \sigma(\lambda) \\
& =\sum_{i=1}^{+\infty} \frac{1}{2^{i}} \int_{\mathbb{T}} V\left(\bigoplus_{i \geq 1} f_{i}\right)(\lambda) E_{i}(\lambda) d \sigma(\lambda)=K V\left(\bigoplus_{i \geq 1} f_{i}\right) .
\end{aligned}
$$

This proves our claim.

This yields a sufficient condition for an operator to have an invariant nondegenerate Gaussian measure.

Proposition 3.19. Let $T$ be a bounded operator on $H$ such that the eigenvectors associated to unimodular eigenvalues span a dense subspace of $H$. Then $T$ has a nondegenerate Gaussian invariant measure.

Proof. For each $i \geq 1$, choose a sequence $\left(\lambda_{n}^{(i)}\right)_{n \geq 0}$ of unimodular eigenvalues such that $\overline{\mathrm{sp}}\left[E_{i}\left(\lambda_{n}^{(i)}\right), i \geq 1, n \geq 0\right]=H$. Let

$$
\sigma=\frac{1}{C} \sum_{i=1}^{+\infty} \frac{1}{2^{i}}\left(\sum_{n=0}^{+\infty} \frac{1}{2^{n}} \delta_{\lambda_{n}^{(i)}}\right),
$$

where $C$ is a normalization constant which turns $\sigma$ into a probability measure. Then $T$ has a $\sigma$-spanning set of eigenvectors associated to unimodular eigenvalues, and the result follows from Proposition 3.18.

Remark 3.20. Suppose that $T$ admits an invariant measure $\mu$ such that

$$
\int_{H}\|z\|^{2} d \mu(z)
$$

is finite and whose support is the whole space. Then $T$ admits a nondegenerate Gaussian invariant measure $m$ : define $S$ on $H$ by the relation

$$
\langle S x, y\rangle=\int_{H}\langle x, z\rangle \overline{\langle y, z\rangle} d \mu(z) \quad \text { for every } x, y \in H .
$$

Since $\mu$ has a moment of order two, $S$ is well defined, self-adjoint, positive and of trace class. Let $m$ be the Gaussian measure whose covariance operator is $S$. Then

$$
\left\langle T S T^{*} x, y\right\rangle=\int_{H}\langle x, z\rangle \overline{\langle y, z\rangle} d(T(\mu))(z)=\langle S x, y\rangle
$$

and $m$ is $T$-invariant. Now if $S x=0,\langle x, z\rangle=0 \mu$-almost surely, hence $x=0: m$ is nondegenerate. Thus the assumption that $m$ is Gaussian is not a restriction here.

Let us conclude this section with an example.

Example 3.21. Let $B$ be the weighted backward shift on $\ell_{2}$ with weights $\left(\omega_{n}\right)_{n \geq 1}$, where $\left(\omega_{n}\right)_{n \geq 1}$ is a bounded sequence of positive real numbers. Suppose that

$$
\liminf _{n \rightarrow+\infty}\left(\omega_{1} \ldots \omega_{n}\right)^{1 / n}>1
$$

and let $\omega_{0}=1$. The canonical basis of $\ell_{2}$ is denoted as usual by $\left(e_{n}\right)_{n \geq 0}$. In this case the unimodular point spectrum of $B$ is simple, $B$ has a perfectly spanning set 
of eigenvectors associated to unimodular eigenvalues, and $\operatorname{ker}(T-\lambda I)$ is spanned by the vector

$$
E(\lambda)=\sum_{n=0}^{+\infty} \frac{\lambda^{n}}{\omega_{0} \ldots \omega_{n}} e_{n}
$$

for $|\lambda|=1$. This makes it easy to determine the covariance operator of the measure $m$ : for every $f \in L^{2}(\mathbb{T}, d \theta)$

$$
K(f)=\sum_{n=0}^{+\infty}\left(\int_{0}^{2 \pi} f\left(e^{i \theta}\right) \frac{e^{i n \theta}}{\omega_{0} \ldots \omega_{n}} \frac{d \theta}{2 \pi}\right) e_{n}
$$

and for every $x \in \ell_{2}$,

Hence

$$
K^{*} x=\sum_{n=0}^{+\infty} \frac{\left\langle x, e_{n}\right\rangle}{\omega_{0} \ldots \omega_{n}} e^{-i n \theta}
$$

$$
S x=K K^{*} x=\sum_{n=0}^{+\infty} \frac{\left\langle x, e_{n}\right\rangle}{\left(\omega_{0} \ldots \omega_{n}\right)^{2}} e_{n},
$$

and $S$ is a diagonal operator with respect to the basis $\left(e_{n}\right)_{n \geq 0}$. Remark that the series

$$
\sum_{n \geq 0} \frac{1}{\left(\omega_{0} \ldots \omega_{n}\right)^{2}}
$$

is convergent, and $S$ is indeed a trace class operator. This means that the Gaussian measure of covariance operator $S$ can be seen as the restriction to $\ell_{2}$ of the product measure defined on $\mathbb{C}^{\mathbb{N}} \simeq\left(\mathbb{R}^{2}\right)^{\mathbb{N}}$ by $\mu=\bigotimes_{n \geq 0} \mu_{n}$, where $\mu_{n}$ is the 2-dimensional centered Gaussian measure on $\mathbb{R}^{2}$ whose covariance matrix is

$$
\left(\begin{array}{cc}
\frac{1}{\left(\omega_{0} \ldots \omega_{n}\right)^{2}} & 0 \\
0 & \frac{1}{\left(\omega_{0} \ldots \omega_{n}\right)^{2}}
\end{array}\right)
$$

3.4. Ergodic properties of measure-preserving operators. Our aim in this section is to obtain conditions implying that an operator $T$ which admits a nondegenerate invariant Gaussian measure is ergodic with respect to this measure. In what follows we will denote by $m$ a Gaussian measure on $H$, and by $\mathcal{B}$ the $\sigma$-algebra of Borel subsets of $H$. Let

$$
T:(H, \mathcal{B}, m) \longrightarrow(H, \mathcal{B}, m)
$$

be a bounded operator which is also a measure-preserving transformation. We denote by $U_{T}$ the associated isometry on $L^{2}(H, \mathcal{B}, m)$ :

$$
\begin{aligned}
U_{T}: L^{2}(H, \mathcal{B}, m) & \longrightarrow L^{2}(H, \mathcal{B}, m) \\
f & \longmapsto[z \mapsto f \circ T(z)] .
\end{aligned}
$$

This is clearly an isometry, but it is not necessarily onto. In order to make $U_{T}$ into a unitary operator, it suffices to change the $\sigma$-algebra on the right-hand side: recall that $\mathcal{B}$ is the $\sigma$-algebra generated by the functions $\Re e\langle., x\rangle: z \mapsto \Re e\langle z, x\rangle$, and consider the $\sigma$-algebra $\mathcal{B}^{\prime}$ generated by the functions $\Re e\left\langle., T^{*} x\right\rangle, x \in H$. Then $\mathcal{B}^{\prime}$ is contained in $\mathcal{B}$ and the functions of the form $z \mapsto P\left(\Re e\left\langle z, T^{*} x_{1}\right\rangle, \ldots, \Re e\left\langle z, T^{*} x_{r}\right\rangle\right)$, where $P$ is a polynomial in $r$ independent variables on $\mathbb{C}^{r}$ and $x_{1}, \ldots, x_{m}$ are $m$ arbitrary vectors of $H$, form a dense subset of $L^{2}\left(H, \mathcal{B}^{\prime}, m\right)([45$, Ch. 8, Theorem 
1.2]). Recall that since $\langle., x\rangle$ is Gaussian, $\langle., x\rangle^{k}$ belongs to $L^{2}(H, \mathcal{B}, m)$ for every $k \geq 0$. This implies that

$$
\begin{aligned}
U_{T}: L^{2}(H, \mathcal{B}, m) & \longrightarrow L^{2}\left(H, \mathcal{B}^{\prime}, m\right) \\
f & \longmapsto f \circ T
\end{aligned}
$$

is a surjective isometry operator. We will not introduce any new notation for this operator, and will specify when needed the $\sigma$-algebras involved. The operator above is in fact the canonical isometry associated to the measure-preserving transformation

$$
T:(H, \mathcal{B}, m) \longrightarrow\left(H, \mathcal{B}^{\prime}, m\right) \text {. }
$$

Our aim is now to prove the following theorem, which gives a sufficient condition for an operator to have a nondegenerate Gaussian invariant measure with respect to which it is weak-mixing.

Theorem 3.22. Suppose that $T$ has a perfectly spanning set of eigenvectors associated to unimodular eigenvalues. There exists a nondegenerate Gaussian invariant measure $m$ on $H$ such that $T:(H, \mathcal{B}, m) \rightarrow(H, \mathcal{B}, m)$ is a weak-mixing transformation.

In order to prove Theorem 3.22 we will use assertion (2) of Definition 3.10, It suffices to prove the convergence for $\mathcal{B}^{\prime}$-measurable functions (if $f$ is $\mathcal{B}$-measurable, then $U_{T} f$ is $\mathcal{B}^{\prime}$-measurable), and moreover there is no loss of generality in assuming that $\langle f, 1\rangle=\langle g, 1\rangle=0$. Thus we are going to show that whenever $f$ and $g$ are $\mathcal{B}^{\prime}$ measurable and $\langle f, 1\rangle=\langle g, 1\rangle=0$,

$$
\lim _{N \rightarrow \infty} \frac{1}{N} \sum_{k=0}^{N-1}\left|\left\langle U_{T}^{k} f, g\right\rangle\right|=0 .
$$

Since the sequence $\left(\left|\left\langle U_{T}^{k} f, g\right\rangle\right|\right)_{k \geq 0}$ is bounded, this is equivalent (see [50, p. 43]) to showing that

$$
\lim _{N \rightarrow \infty} \frac{1}{N} \sum_{k=0}^{N-1}\left|\left\langle U_{T}^{k} f, g\right\rangle\right|^{2}=0 .
$$

Remark that all the functions $f_{x}=\langle., x\rangle: z \mapsto\langle z, x\rangle$ belong to $L^{2}(H, \mathcal{B}, m)$ since $m$ has a moment of order two, and that these functions have zero mean. We first consider the special case where $f$ and $g$ are one of these functions $f_{x}$.

Lemma 3.23. For any $x$ and $y$ in $H$,

$$
\lim _{N \rightarrow \infty} \frac{1}{N} \sum_{k=0}^{N-1}\left|\left\langle U_{T}^{k} f_{x}, f_{y}\right\rangle\right|^{2}=0 .
$$

Proof. Let $\sigma$ be the measure given in Definition 3.1 let $K$ and $V$ be as in the proof of Proposition 3.18, and let $S=K^{*} K$. We have

$$
\left\langle U_{T}^{k} f_{x}, f_{y}\right\rangle=\int_{H}\left\langle T^{k} z, x\right\rangle \overline{\langle z, y\rangle} d m(z)=\left\langle T^{k} S y, x\right\rangle .
$$

But $S=K K^{*}$ with $K^{*} T^{*}=V^{*} K^{*}$, hence $\left\langle U_{T}^{k} f_{x}, f_{y}\right\rangle=\left\langle V^{k} K^{*} y, K^{*} x\right\rangle$. Now $V$ acts on $\bigoplus_{i \geq 1} L^{2}(\mathbb{T}, \sigma)$ as multiplication by $\lambda$ on each component. Writing $K^{*} x$ 
as $\left(f_{i}\right)_{i \geq 1}$ and $K^{*} y$ as $\left(g_{i}\right)_{i \geq 1}$ with $f_{i}$ and $g_{i}$ in $L^{2}(\mathbb{T}, \sigma)$ and $\sum_{i \geq 1}\left\|f_{i}\right\|^{2}<+\infty$, $\sum_{i \geq 1}\left\|g_{i}\right\|^{2}<+\infty$ yields that

$$
\left\langle U_{T}^{k} f_{x}, f_{y}\right\rangle=\sum_{i=1}^{+\infty} \int_{\mathbb{T}} \lambda^{k} g_{i}(\lambda) \overline{f_{i}(\lambda)} d \sigma(\lambda)=\int_{\mathbb{T}} \lambda^{k} \sum_{i=1}^{+\infty} g_{i}(\lambda) \overline{f_{i}(\lambda)} d \sigma(\lambda),
$$

and the sequence $\left(\left\langle U_{T}^{k} f_{x}, f_{y}\right\rangle\right)$ appears as the negative half of the sequence of Fourier coefficients of the measure

$$
d \mu(\lambda)=\sum_{i=1}^{+\infty} g_{i}(\lambda) \overline{f_{i}(\lambda)} d \sigma(\lambda)
$$

Since $\sigma$ is continuous, $\mu$ is also continuous, and Wiener's theorem (see [36, p. 42]) implies that the Cesaro mean

$$
\frac{1}{N} \sum_{k=0}^{N-1}\left|\left\langle U_{T}^{k} f_{x}, f_{y}\right\rangle\right|^{2}=0
$$

goes to zero as $n$ goes to infinity.

Now consider the following two closed subspaces of $L^{2}(H, \mathcal{B}, m)$ and $L^{2}\left(H, \mathcal{B}^{\prime}, m\right)$ respectively:

$$
\begin{gathered}
\mathcal{G}=\overline{\mathrm{sp}}^{L^{2}(H, \mathcal{B}, m)}[\langle., x\rangle ; x \in H], \\
\mathcal{G}^{\prime}=\overline{\mathrm{sp}}^{L^{2}\left(H, \mathcal{B}^{\prime}, m\right)}\left[\left\langle., T^{*} x\right\rangle ; x \in H\right] .
\end{gathered}
$$

These subspaces are Gaussian subspaces in the sense that any function in $\mathcal{G}$ or $\mathcal{G}^{\prime}$ has symmetric complex Gaussian distribution: indeed this is the case for any function $\langle., x\rangle$ since the measure $m$ is centered Gaussian, and the norm-closure of a space of Gaussian functions is also Gaussian. The following lemma follows directly from Lemma 3.23 and some straightforward computations.

Lemma 3.24. For any functions $f$ and $g$ in $\mathcal{G}$,

$$
\lim _{n \rightarrow \infty} \frac{1}{N} \sum_{k=0}^{N-1}\left|\left\langle U_{T}^{k} f, g\right\rangle\right|^{2}=0
$$

The theory of Fock spaces then allows us to extend this property to general functions $f$ and $g$ in $\mathcal{B}^{\prime}$. Here we recall the definitions and facts about Fock spaces that will be of use in the sequel; for a thorough account see [45, Ch. 8] or [35].

Since $\mathcal{B}$ (resp. $\mathcal{B}^{\prime}$ ) is the $\sigma$-algebra generated by the functions in $\mathcal{G}$ (resp. $\mathcal{G}^{\prime}$ ), applying the Weierstrass Theorem gives that ([45, Ch 8, Theorem 1.2])

$$
\begin{gathered}
L^{2}(H, \mathcal{B}, m)=\overline{\mathrm{sp}}^{L^{2}(H, \mathcal{B}, m)}\left[g^{k} ; g \in \mathcal{G}, k \geq 0\right], \\
L^{2}\left(H, \mathcal{B}^{\prime}, m\right)=\overline{\mathrm{sp}}^{L^{2}\left(H, \mathcal{B}^{\prime}, m\right)}\left[g^{\prime k} ; g^{\prime} \in \mathcal{G}^{\prime}, k \geq 0\right] .
\end{gathered}
$$

Let $\mathcal{G}^{n}$ denote the space of homogeneous polynomials of degree $n$ of elements of $\mathcal{G}$, with $\mathcal{G}^{0}=\mathbb{C}$. Then the spaces $\mathcal{G}^{n}, n \geq 0$, are linearly independent ([45, Ch. 8, Lemma 2.3]), and the Wick transform orthonormalizes these subspaces.

Definition 3.25. The Wick transform is defined on the spaces $\mathcal{G}^{n}$ in the following way:

(1) if $f$ is constant, $: f:=f$,

(2) if $f \in \mathcal{G}^{n}, n \geq 1,: f:=f-\mathcal{P}_{n} f$. 
Here $\mathcal{P}_{n}$ denotes the orthogonal projection onto $\overline{\mathrm{sp}}^{L^{2}(H, \mathcal{B}, m)}\left[\mathcal{G}^{k} ; 0 \leq k \leq n-1\right]$.

Hence we have

$$
L^{2}(H, \mathcal{B}, m)=\bigoplus_{k \geq 0}: \mathcal{G}^{k}: \quad \text { and } \quad L^{2}\left(H, \mathcal{B}^{\prime}, m\right)=\bigoplus_{k \geq 0}: \mathcal{G}^{\prime k}:
$$

where the sums above are orthogonal direct sums. This decomposition allows one to identify $L^{2}(H, \mathcal{B}, m)\left(\right.$ resp. $\left.L^{2}\left(H, \mathcal{B}^{\prime}, m\right)\right)$ with the Fock space $\mathcal{F}(\mathcal{G})\left(\right.$ resp. $\left.\mathcal{F}\left(\mathcal{G}^{\prime}\right)\right)$, which we now define.

On the Hilbert tensor product $\bigotimes_{n} \mathcal{G}$, consider the scalar product $\langle., .\rangle_{\otimes}$ defined on elementary tensors by

$$
\left\langle g_{1} \otimes \ldots \otimes g_{n}, h_{1} \otimes \ldots \otimes h_{n}\right\rangle_{\otimes}=\left\langle g_{1}, h_{1}\right\rangle \ldots\left\langle g_{n}, h_{n}\right\rangle \quad \text { for } g_{i}, h_{i} \in \mathcal{G} .
$$

The space $\mathcal{G}_{\odot}^{n}$ is the range of the projection

$$
\begin{aligned}
\text { Sym }: \bigotimes_{n} \mathcal{G} & \longrightarrow \mathcal{G}_{\odot}^{n}, \\
f_{1} \otimes \ldots \otimes f_{n} & \longmapsto \frac{1}{n !} \sum_{\tau \in \Sigma_{n}} f_{\tau_{1}} \otimes \ldots \otimes f_{\tau_{n}} .
\end{aligned}
$$

Then

$$
\left\langle\operatorname{Sym}\left(g_{1} \otimes \ldots \otimes g_{n}\right), \operatorname{Sym}\left(h_{1} \otimes \ldots \otimes h_{n}\right)\right\rangle_{\otimes}=\frac{1}{n !} \sum_{\tau \in \Sigma_{n}}\left\langle g_{\tau_{1}}, h_{1}\right\rangle \ldots\left\langle g_{\tau_{n}}, h_{n}\right\rangle .
$$

For $f$ and $g$ in $\mathcal{G}_{\odot}^{n}$, the symmetric scalar product $\langle., .\rangle_{\odot}$ is defined by the formula

$$
\langle f, g\rangle_{\odot}=n !\langle f, g\rangle_{\otimes}
$$

Definition 3.26. The Fock space $\mathcal{F}(\mathcal{G})$ over $\mathcal{G}$ is

$$
\mathcal{F}(\mathcal{G})=\bigoplus_{n \geq 0} \mathcal{G}_{\odot}^{n},
$$

where the sum is an orthogonal direct sum and each $\mathcal{G}_{\odot}^{n}$ is endowed with the scalar product $\langle., .\rangle_{\odot}$.

One of the interests of this is that $L^{2}(H, \mathcal{B}, m)$ can be identified with $\mathcal{F}(\mathcal{G})$ thanks to the map

$$
\begin{aligned}
: \mathcal{G}^{n} & : \longrightarrow \mathcal{G}_{\odot}^{n} \\
: f_{1} \ldots f_{n}: & \longmapsto \operatorname{Sym}\left(f_{1} \otimes \ldots \otimes f_{n}\right),
\end{aligned}
$$

which extends uniquely to an isometry of $: \mathcal{G}^{n}:$ onto $\mathcal{G}_{\odot}^{n}$. Since

$$
L^{2}(H, \mathcal{B}, m)=\bigoplus_{k \geq 0}: \mathcal{G}^{k}:
$$

this yields that $L^{2}(H, \mathcal{B}, m)=\mathcal{F}(\mathcal{G})$ and also that $L^{2}\left(H, \mathcal{B}^{\prime}, m\right)=\mathcal{F}\left(\mathcal{G}^{\prime}\right)$.

Definition 3.27. If $A$ is any contraction from $\mathcal{G}$ into $\mathcal{G}^{\prime}$, it is possible to define its Fock power $\mathcal{F}(A)$ from $\mathcal{F}(\mathcal{G})$ into $\mathcal{F}\left(\mathcal{G}^{\prime}\right)$ : the $n^{\text {th }}$ tensor product $\bigotimes_{n} A$ of $A$ acts on $\bigotimes_{n} \mathcal{G}$ and maps $\bigotimes_{n} \mathcal{G}$ into $\bigotimes_{n} \mathcal{G}^{\prime}$ by the formula

$$
\left(\bigotimes_{n} A\right)\left(f_{1} \otimes \ldots \otimes f_{n}\right)=A f_{1} \otimes \ldots \otimes A f_{n}
$$

Now $\bigotimes_{n} A$ maps symmetric tensor products on symmetric tensor products, and this makes it possible to consider the restriction of $\mathcal{F}(A)$ to $\mathcal{G}_{\odot}^{n}$. Defining $\mathcal{F}(A)$ on 
$\mathcal{G}_{\odot}^{n}$ to be this restriction, $\mathcal{F}(A)$ extends to a contraction on $\mathcal{F}(\mathcal{G})$ into $\mathcal{F}\left(\mathcal{G}^{\prime}\right)$, which is the Fock power of $A$.

We now come back to our main problem: recall that our measure-preserving transformation $T$ induces a surjective isometry operator $U_{T}: L^{2}(H, \mathcal{B}, m) \rightarrow$ $L^{2}\left(H, \mathcal{B}^{\prime}, m\right)$, and that moreover $U_{T}(\mathcal{G})=\mathcal{G}^{\prime}$.

Lemma 3.28. The operator $U_{T}: L^{2}(H, \mathcal{B}, m) \rightarrow L^{2}\left(H, \mathcal{B}^{\prime}, m\right)$ can be identified to the Fock power of its restriction to $\mathcal{G}$ via the isometry

$$
: f_{1} \ldots f_{n}: \longmapsto \operatorname{Sym}\left(f_{1} \otimes \ldots \otimes f_{n}\right)
$$

of : $\mathcal{G}^{n}$ : onto $\mathcal{G}_{\odot}^{n}$.

Proof. Denote by $V_{T}$ the restriction of $U_{T}$ to $\mathcal{G}: V_{T}(\mathcal{G})=\mathcal{G}^{\prime}$. Then

$$
\mathcal{F}\left(V_{T}\right)\left(\operatorname{Sym}\left(f_{1} \otimes \ldots \otimes f_{n}\right)\right)=\operatorname{Sym}\left(U_{T} f_{1} \otimes \ldots \otimes U_{T} f_{n}\right)
$$

hence we need to show that for every $n$-tuple $f_{1}, \ldots, f_{n}$ of elements of $\mathcal{G}$,

$$
U_{T}\left(: f_{1} \ldots f_{n}:\right)=: U_{T} f_{1} \ldots U_{T} f_{n}:
$$

(and this will imply that $U_{T}\left(: \mathcal{G}^{n}:\right)=: \mathcal{G}^{\prime n}:$ ). Recall that

$$
: f_{1} \ldots f_{n}:=f_{1} \ldots f_{n}-\mathcal{P}_{n}\left(f_{1} \ldots f_{n}\right)
$$

where $\mathcal{P}_{n}$ denotes the projection onto $\overline{\mathrm{sp}}^{L^{2}(H, \mathcal{B}, m)}\left[\mathcal{G}^{k} ; 0 \leq k \leq n-1\right]$. In the same way, $\mathcal{P}_{n}^{\prime}$ denotes the orthogonal projection onto

$$
\overline{\mathrm{sp}}^{L^{2}(H, \mathcal{B}, m)}\left[\mathcal{G}^{\prime k} ; 0 \leq k \leq n-1\right] .
$$

Hence we have to show that $U_{T}\left(\mathcal{P}_{n}\right)\left(f_{1} \ldots f_{n}\right)=\mathcal{P}_{n}^{\prime}\left(U_{T}\left(f_{1} \ldots f_{n}\right)\right)$. Now $\mathcal{P}_{n}^{\prime}\left(U_{T}\left(f_{1} \ldots f_{n}\right)\right)$ is characterized by the two facts that $\mathcal{P}_{n}^{\prime}\left(U_{T}\left(f_{1} \ldots f_{n}\right)\right)$ is in $\overline{\mathrm{sp}}^{L^{2}(H, \mathcal{B}, m)}\left[\mathcal{G}^{\prime k} ; 0 \leq k \leq n-1\right]$ and

$$
\left\langle\mathcal{P}_{n}^{\prime}\left(U_{T}\left(f_{1} \ldots f_{n}\right)\right), h_{1}^{\prime} \ldots h_{k}^{\prime}\right\rangle_{\odot}=\left\langle\left(U_{T}\left(f_{1} \ldots f_{n}\right)\right), h_{1}^{\prime} \ldots h_{k}^{\prime}\right\rangle_{\odot}
$$

for any $h_{1}^{\prime} \ldots h_{k}^{\prime}$ in $\mathcal{G}^{\prime}$. Now $\mathcal{P}_{n}^{\prime}\left(U_{T}\left(f_{1} \ldots f_{n}\right)\right)$ is clearly in

$$
\overline{\mathrm{sp}}^{L^{2}(H, \mathcal{B}, m)}\left[\mathcal{G}^{\prime k} ; 0 \leq k \leq n-1\right]
$$

and

$$
\begin{aligned}
\left\langle U_{T}\left(\mathcal{P}_{n}\left(f_{1} \ldots f_{n}\right)\right), h_{1}^{\prime} \ldots h_{k}^{\prime}\right\rangle_{\odot} & =\left\langle\mathcal{P}_{n}\left(f_{1} \ldots f_{n}\right), U_{T}^{-1}\left(h_{1}^{\prime} \ldots h_{k}^{\prime}\right)\right\rangle_{\odot} \\
& =\left\langle f_{1} \ldots f_{n}, U_{T}^{-1}\left(h_{1}^{\prime} \ldots h_{k}^{\prime}\right)\right\rangle_{\odot} \\
& =\left\langle U_{T}\left(f_{1} \ldots f_{n}\right), h_{1}^{\prime} \ldots h_{k}^{\prime}\right\rangle_{\odot} .
\end{aligned}
$$

Thus $U_{T}: L^{2}(H, \mathcal{B}, m) \rightarrow L^{2}\left(H, \mathcal{B}^{\prime}, m\right)$ can be identified to $\mathcal{F}\left(V_{T}\right): \mathcal{F}(\mathcal{G}) \rightarrow$ $\mathcal{F}\left(\mathcal{G}^{\prime}\right)$.

Proof of Theorem 3.22. Let $f_{1}, \ldots, f_{r}, g_{1}, \ldots, g_{s}$ be elements of $\mathcal{G}$. Let us show that the Cesaro mean

$$
\frac{1}{N} \sum_{k=0}^{N-1}\left|\left\langle U_{T}^{k}\left(: f_{1} \ldots f_{r}:\right),: g_{1} \ldots g_{s}:\right\rangle\right|
$$

goes to zero as $N$ goes to infinity. By Lemma 3.28, this quantity is equal to

$$
\frac{1}{N} \sum_{k=0}^{N-1}\left|\left\langle: U_{T}^{k}\left(f_{1} \ldots f_{r}\right):,: g_{1} \ldots g_{s}:\right\rangle\right| \text {. }
$$


If $r \neq s$, this quantity is 0 . If $r=s$, it is equal to

$$
\begin{gathered}
\frac{1}{N} \sum_{k=0}^{N-1}\left|\left\langle\operatorname{Sym}\left(U_{T}^{k} f_{1} \otimes \ldots U_{T}^{k} f_{r}\right), \operatorname{Sym}\left(g_{1} \otimes \ldots \otimes g_{r}\right)\right\rangle_{\odot}\right| \\
\quad=\frac{1}{N} \sum_{k=0}^{N-1}\left|\sum_{\tau \in \Sigma_{r}}\left\langle U_{T}^{k} f_{\tau(1)}, g_{1}\right\rangle \ldots\left\langle U_{T}^{k} f_{\tau(r)}, g_{r}\right\rangle\right| \\
\leq \sum_{\tau \in \Sigma_{r}}\left(\frac{1}{N} \sum_{k=0}^{N-1}\left|\left\langle U_{T}^{k} f_{\tau(1)}, g_{1}\right\rangle \ldots\left\langle U_{T}^{k} f_{\tau(r)}, g_{r}\right\rangle\right|\right) \\
\leq C \sum_{\tau \in \Sigma_{r}}\left(\frac{1}{N} \sum_{k=0}^{N-1}\left|\left\langle U_{T}^{k} f_{\tau(1)}, g_{1}\right\rangle\right|\right)
\end{gathered}
$$

for some positive constant $C$. Lemma 3.24 implies that the limit of this last sum is 0 . Since

$$
L^{2}(H, \mathcal{B}, m)=\bigoplus_{k \geq 0}: \mathcal{G}^{k}:
$$

this finally yields that $T$ is weak-mixing with respect to $m$.

The conclusion of Theorem 3.22 can be strengthened to give strong-mixing transformations if we impose an additional restriction on the measure $\sigma$.

Theorem 3.29. Suppose that $T$ has a $\sigma$-spanning set of eigenvectors associated to unimodular eigenvalues, with $\sigma$ a Rajchman measure, which means that the Fourier coefficients of the measure $\sigma$ tend to zero as $|n|$ goes to infinity (this is the case in particular if $\sigma$ is absolutely continuous with respect to the Lebesgue length measure on $\mathbb{T})$. Then $T$ admits a nondegenerate Gaussian invariant measure such that $T:(H, \mathcal{B}, m) \longrightarrow(H, \mathcal{B}, m)$ is strong-mixing.

Proof. We retain the notation of the previous theorem. Our goal is now to show that for any pair $(f, g)$ of $\mathcal{B}^{\prime}$-measurable functions with $\langle f, 1\rangle=\langle g, 1\rangle=0,\left\langle U_{T}^{N} f, g\right\rangle$ tends to zero. For any $x$ and $y$ in $H,\left\langle U_{T}^{N} f_{x}, f_{y}\right\rangle$ is the $(-N)^{t h}$ Fourier coefficient of a measure which is absolutely continuous with respect to $\sigma$. But any measure which is absolutely continuous with respect to a Rajchman measure is a Rajchman measure, too (see for instance [37, p. 77]). Hence $\left\langle U_{T}^{N} f_{x}, f_{y}\right\rangle$ tends to zero. The rest of the proof runs exactly along the same lines, and just as before the concluding argument is that if $f_{1}, \ldots, f_{n}$ are $n$ elements of $\mathcal{G}$ and $\tau \in \Sigma_{n},\left\langle U_{T}^{k} f_{\tau(1)}, g_{1}\right\rangle \ldots\left\langle U_{T}^{k} f_{\tau(n)}, g_{n}\right\rangle$ goes to 0 as $N$ goes to infinity.

We are now ready to prove Theorem 3.2 .

Proof of Theorem 3.2. The first point follows directly from Theorem 3.22 and Proposition 3.12 . The proof of the second assertion of Theorem 3.2 uses the weakmixing property of $T$ : for any pair $(A, B)$ of measurable subsets of $H$, there exists a sequence $\left(n_{k}\right)$ of density 1 such that $m\left(T^{-n_{k}}(A) \cap B\right) \rightarrow m(A) m(B)([50$, p. 43]). Applying this to a pair $(U, V)$ of nonempty open subsets of $H$ implies in particular that $T^{-n_{k}}(U) \cap V$ is nonempty for a sequence $\left(n_{k}\right)$ of density 1 .

When $T$ satisfies the assumptions of Theorem 3.29, $T$ is even topologically mixing: choose a measure $m$ with respect to which $T$ is strong-mixing. Then for any measurable sets $A$ and $B, m\left(T^{-n}(A) \cap B\right) \rightarrow m(A) m(B)$, hence for any pair $(U, V)$ 
of nonempty open sets, there exists an integer $n_{0}$ such that $T^{-n}(U) \cap V$ is nonempty for every $n \geq n_{0}$.

Remark 3.30. That the operator $K$ which appears in the equation $T K=K V$ is Hilbert-Schmidt, is crucial, and cannot be replaced by a weaker compactness assumption: if we suppose merely that $K$ is compact (and that $V^{n}$ tends weakly to zero as $|n|$ goes to infinity for instance), then $T$ is still hypercyclic ([5], see also [4] for a simplified proof in a special case), but not necessarily frequently hypercyclic. This happens for instance for the backward shift $B$ on the Bergman space $A^{2}: B$ satisfies the equation $T K=K V$ with $V$ the multiplication operator by the independent variable $\bar{\lambda}$ on $L^{2}\left(\mathbb{T}, \frac{d \theta}{2 \pi}\right)$ and $K: L^{2} \rightarrow A^{2}$ the complex Riesz projection defined by $K f(z)=\sum_{n=0}^{+\infty} \hat{f}(n) z^{n}$. Then $K$ is compact and has dense range, but is not Hilbert-Schmidt. This is coherent with the fact that $B$ is hypercyclic without being frequently hypercyclic. The idea of using equation $(F)$ to prove the hypercyclicity of an operator was introduced by Bourdon and Shapiro in [14, where they investigate the hypercyclicity properties of functions $\phi(B)$ of the Bergman backward shift, and show that $B$ satisfies equation $(F)$.

Remark 3.31. We can now give a kind of negative answer to something we suggested in the Introduction: the Hypercyclicity Criterion Problem is not clearly related to the ergodic-theoretic statement that if $T$ is a measure-preserving transformation on a measure space $(X, \mathcal{B}, \mu), T \times T$ is ergodic on $(X \times X, \mathcal{B} \otimes \mathcal{B}, \mu \otimes \mu)$ if and only if $T$ is weak-mixing: an operator which is not frequently hypercyclic cannot be ergodic with respect to an invariant measure $\mu$ on $H$ whose support is the whole space. It was conceivable to think that both problems were in some sense related, but this seems to point out that the situation is much more involved.

\section{Properties of Frequently hypercyclic operators}

As we already mentioned in the Introduction, our purpose in this section is to understand better the difference between the notions of hypercyclicity and frequent hypercyclicity, the main difference being the lack of Baire Category arguments in hypercyclicity results. Recall that as soon as $T$ is hypercyclic, the set of hypercyclic vectors for $T$ can be written as

$$
H C(T)=\bigcap_{k \geq 1} \bigcup_{n \geq 0}\left\{x \in X ; T^{n} x \in B_{k}\right\},
$$

where $\left(B_{k}\right)_{k \geq 1}$ is a countable basis of open sets of $X$. Hence $H C(T)$ is a dense $G_{\delta}$ subset of $X$. This breaks down for frequently hypercyclic vectors.

Proposition 4.1. Let $T$ be a bounded operator on a Banach space $X$. Suppose that one of the two following conditions is satisfied:

- there exists a dense subset $X_{0}$ of $X$ with $T^{n} x \rightarrow 0$ for any $x \in X_{0}$,

- there exists a dense subset $Y_{0}$ of $X$ with $\left\|T^{n} x\right\| \rightarrow+\infty$ for any $x \in Y_{0}$.

Then $F H C(T)$ is not a residual set in $X$.

In particular, this is true for all concrete frequently hypercyclic operators (backward shifts, adjoints of multipliers, composition operators) considered in this paper. By a result of Müller ([41]), the second condition is fulfilled as soon as the spectral radius $r(T)$ of $T$ satisfies $r(T)>1$. 
Proof. We just consider the case where the first condition is satisfied. The proof for the other case is exactly the same, provided we replace the inequalities $<1 / 2$ by inequalities $>2$. By contradiction, suppose that $F H C(T)$ contains a set $\bigcap_{k \geq 1} \Omega_{k}$, where the $\Omega_{k}$ 's are dense open subsets of $X$. We construct by induction a sequence $\left(w_{k}\right)_{k \geq 1}$ of vectors of $X$, a sequence $\left(\rho_{k}\right)_{k \geq 1}$ of positive real numbers and an increasing sequence of integers $\left(N_{k}\right)_{k \geq 1}$ such that:

(1) $\left(\rho_{k}\right)_{k \geq 1}$ decreases to zero.

(2) $B\left(w_{k}, \rho_{k}\right) \subseteq B\left(w_{k-1}, \rho_{k-1}\right)$.

(3) $B\left(w_{k}, \rho_{k}\right) \subseteq \Omega_{k}$.

(4) For each $w$ in $B\left(w_{k}, \rho_{k}\right)$, and for each $n \in\left[N_{k}, k N_{k}\right],\left\|T^{n} w\right\|<1 / 2$.

Step 1: Let $z_{1}$ belong to $\Omega_{1}$, and let $r_{1}>0$ be such that $B\left(z_{1}, r_{1}\right) \subseteq \Omega_{1}$. By density of $X_{0}$, one may find $w_{1}$ in $B\left(z_{1}, r_{1}\right)$ and $N_{1} \geq 0$ such that $n \geq N_{1}$ implies $\left\|T^{n} w\right\|<1 / 2$. By continuity of $T^{N_{1}}$, if $\rho_{1}$ is chosen small enough (one should impose $\left.B\left(w_{1}, \rho_{1}\right) \subset B\left(z_{1}, r_{1}\right)\right)$, one has $\left\|T^{N_{1}} w\right\|<1 / 2$ for any $w \in B\left(w_{1}, \rho_{1}\right)$.

Step $k$ : Choose $z_{k}$ in $\Omega_{k} \cap B\left(w_{k-1}, \rho_{k-1}\right)$, and $r_{k}>0$ such that $B\left(z_{k}, r_{k}\right)$ is contained in $\Omega_{k} \cap B\left(w_{k-1}, \rho_{k-1}\right)$. As previously, one may consider $w_{k}$ in the ball $B\left(z_{k}, r_{k}\right)$ and $N_{k} \geq N_{k-1}$ such that $n \geq N_{k}$ implies $\left\|T^{n} w\right\|<1 / 2$. By continuity of $T^{N_{k}}, \ldots, T^{\bar{k} N_{k}}$, one may adjust $\rho_{k}<\min \left(\rho_{k-1}, 1 / k\right)$ so that it satisfies $B\left(w_{k}, \rho_{k}\right) \subset B\left(z_{k}, r_{k}\right)$ and, if $w$ belongs to $B\left(w_{k}, \rho_{k}\right)$ and $n$ belongs to $\left[N_{k}, k N_{k}\right]$, then $\left\|T^{n} w\right\|<1 / 2$.

Now take $w$ as the limit of $\left(w_{k}\right)$, which belongs to $\bigcap_{k \geq 1} \Omega_{k}$, and let $e$ be any unit vector of $X$. Observe that, for any $k \geq 1$ and any $n \in\left[N_{k}, k N_{k}\right]$, one has $\left\|T^{n} w\right\| \leq 1 / 2$. Therefore

$$
\frac{1}{k N_{k}} \#\left\{n \leq k N_{k} ; n \in A p p(T, w, e, 1 / 4)\right\} \leq \frac{1}{k},
$$

and letting $k$ go to infinity, we get that the lower density of $\operatorname{App}(T, w, e, 1 / 4)$ is 0 . Hence $w$ is not a frequently hypercyclic vector, and this contradicts the initial assumption that $\bigcap_{k \geq 1} \Omega_{k} \subseteq F H C(T)$.

But there is a linear property of the set of hypercyclic vectors, whose proof does not involve any category argument, which remains true for frequently hypercyclic operators.

Proposition 4.2. Let $T$ be a frequently hypercyclic operator on a separable $\mathcal{F}$-space $X$. There is a dense invariant manifold $M$ of $X$, every nonzero vector of which is frequently hypercyclic for $T$.

Proof. The proof is patterned after Bourdon's argument in [13. Let $x$ be a frequently hypercyclic vector, and set $M=\{P(T)(x), P \in \mathbb{K}[\xi]\}$. Every nonzero vector of $M$ is frequently hypercyclic for $T$. Let $y=P(T)(x)$ be such a vector with $P \neq 0$. Then $P(T)$ has dense range ([13, , 9], [51]). Fix any $f$ in $X$, and $\varepsilon>0$, and choose $g \in X$ such that $\|P(T)(g)-f\|<\varepsilon / 2$. Now if $n \in A p p(T, x, g, \varepsilon /(2\|P(T)\|))$, then

$$
\begin{aligned}
\left\|T^{n}(y)-f\right\| & \leq\left\|P(T)\left(T^{n} x\right)-P(T)(g)\right\|+\|P(T)(g)-f\| \\
& <\|P(T)\|\left\|T^{n} x-g\right\|+\frac{\varepsilon}{2} \leq \varepsilon .
\end{aligned}
$$

This proves that $n$ belongs to $\operatorname{App}(T, y, f, \varepsilon)$, and therefore this last set has positive lower density: $y$ belongs to $F H C(T)$. 
The lack of residuality properties makes it sometimes difficult to prove results for frequently hypercyclic operators which are obviously true for hypercyclic operators: for instance it is immediate that if $T$ is a hypercyclic invertible operator, $T^{-1}$ is also hypercyclic. But we do not know the answer to the following question.

Question 4.3. Let $T$ be an invertible frequently hypercyclic operator. Is $T^{-1}$ frequently hypercyclic? What can be said about $F H C\left(T^{-1}\right)$ ?

The same drawback appears when dealing with problems of common frequent hypercyclicity: if $\left(T_{k}\right)_{k \geq 1}$ is a sequence of hypercyclic operators, $\bigcap_{k \geq 1} H C\left(T_{k}\right)$ is a dense $G_{\delta}$ subset of $X$, in particular it is nonempty, and the $T_{k}$ 's have a common hypercyclic vector (and even a common dense linear manifold consisting, except for 0 , of hypercyclic vectors $([28))$. But it is unclear whether or not this extends to frequently hypercyclic operators.

Question 4.4. Let $\left(T_{k}\right)_{k \geq 1}$ be a sequence of frequently hypercyclic operators. Do the $T_{k}$ 's have a common frequently hypercyclic vector? Do they have a common dense linear manifold of frequently hypercyclic vectors?

If $T$ is an operator on a Hilbert space which satisfies the assumptions of Theorem 3.22 and $m$ is the Gaussian measure with respect to which $T$ is weak-mixing, then $m(F H C(T))=1$, thus $F H C(T)$ is big in a measure-theoretic sense. In this case it is easy to see that Question 4.3 has a positive answer: if $T$ is invertible and has a perfectly spanning set of eigenvectors associated to unimodular eigenvalues, it is obvious that the same property holds true for $T^{-1}$. But the situation is already more intricate for Question 4.4, even for two operators: if $T_{1}$ and $T_{2}$ are two operators satisfying the assumptions of Theorem 3.22 , and $m_{1}$ and $m_{2}$ the two Gaussian measures involved, then $m_{1}\left(F H C\left(T_{1}\right)\right)=m_{2}\left(F H C\left(T_{2}\right)\right)=1$, but it may happen that $m_{1}$ and $m_{2}$ are not absolutely continuous with respect to each other. Let $\left(\alpha_{n}\right)_{n \geq 1}$ be a sequence of positive numbers such that

$$
\varepsilon \leq \limsup _{n \rightarrow+\infty} \alpha_{n}{ }^{\frac{1}{n}}<1
$$

for some positive $\varepsilon$, and consider the weighted backward shift $B_{\alpha}$ on $\ell_{2}$ with weights $\left(\gamma_{n}\right)_{n \geq 1}$ defined by $\gamma_{1}=1$ and $\gamma_{n}=\frac{\alpha_{n-1}}{\alpha_{n}}$ for $n \geq 1$. Then

$$
\frac{1}{\gamma_{1} \ldots \gamma_{n}}=\alpha_{n},
$$

and we are exactly in the situation of Example $3.21 B_{\alpha}$ is weak-mixing with respect to $m_{\alpha}$, which can be identified via the unitary operator

$$
\begin{aligned}
U: \ell_{2} & \longrightarrow \mathbb{C}^{\mathbb{N}} \\
x & \longmapsto\left(\left\langle x, e_{1}\right\rangle, \ldots,\left\langle x, e_{n}\right\rangle, \ldots\right)
\end{aligned}
$$

to the product measure $\mu_{\alpha}$ on $\mathbb{C}^{\mathbb{N}}, \mu_{\alpha}=\bigotimes_{n \geq 0} \mu_{n, \alpha}$, where $\mu_{n, \alpha}$ is the 2 -dimensional centered Gaussian measure on $\mathbb{R}^{2}$ whose covariance matrix is

$$
\left(\begin{array}{cc}
\alpha_{n} & 0 \\
0 & \alpha_{n}
\end{array}\right) .
$$

Now if $\left(\alpha_{n}\right)_{n \geq 1}$ and $\left(\beta_{n}\right)_{n \geq 1}$ are two such sequences and $B_{\alpha}$ and $B_{\beta}$ the associated weighted shifts, the same computation as in [12, Example 2.7.6.] shows that $m_{\alpha}$ 
and $m_{\beta}$ are equivalent if and only if the product

$$
\prod_{n=1}^{+\infty} \frac{2 \sqrt{\alpha_{n} \beta_{n}}}{\alpha_{n}+\beta_{n}}
$$

is convergent, which is in turn equivalent to the condition that

$$
\sum_{n=1}^{+\infty}\left(\sqrt{\frac{\alpha_{n}}{\beta_{n}}}-1\right)^{2}
$$

is finite. If we choose $\alpha_{n}=2^{-n}$ and $\beta_{n}=4^{-n}$, this condition is not satisfied, and hence $m_{\alpha}$ and $m_{\beta}$ are orthogonal measures (two Gaussian measures are either equivalent or mutually singular). Thus is does not follow that $B_{\alpha}$ and $B_{\beta}$, which are both frequently hypercyclic, have a common frequently hypercyclic vector.

Questions of common hypercyclicity have also been considered for some uncountable families $\left(T_{\lambda}\right)_{\lambda \in I}$ of hypercyclic operators. Answering a question of Salas (48), Abakumov and Gordon have given in [1] the first positive result: the set $\bigcap_{\lambda>1} H C(\lambda B)$, where $B$ is the backward shift acting on $\ell_{2}$, is nonempty. It is proved in [16] that the same is true for $\bigcap_{a>0} H C\left(T_{a}\right)$, where $T_{a}$ is the translation operator $f \mapsto[z \mapsto f(z+a)]$ on $\mathcal{H}(\mathbb{C})$. Since each operator $\lambda B$ or $T_{a}$ is individually frequently hypercyclic, a natural question arises. Does there exist a common frequently hypercyclic vector for the family $(\lambda B)_{\lambda>1}$ ? for the family $\left(T_{a}\right)_{a>0}$ ? The answer is rather surprising.

Theorem 4.5. Let $B$ be the backward shift acting on $\ell_{2}$, and $\Lambda$ an uncountable subset of $] 1,+\infty[$. Then the set of common frequently hypercyclic vectors for the family $(\lambda B)_{\lambda \in \Lambda}$ is empty.

Theorem 4.6. For $a>0$, let $T_{a}$ be the translation operator $f \mapsto[z \mapsto f(z+a)]$ on $\mathcal{H}(\mathbb{C})$. Then the set of common frequently hypercyclic vectors for the family $\left(T_{a}\right)_{a>0}$ is nonempty.

Proof of Theorem 4.5. By contradiction, suppose that

$$
\bigcap_{\lambda \in \Lambda} F H C(\lambda B)
$$

is nonempty. Then it contains a common frequently hypercyclic vector $x=\left(x_{n}\right)_{n \geq 0}$. To each $\lambda \in \Lambda$, we associate the positive number

$$
\delta_{\lambda}=\underline{\text { dens }} A p p\left(\lambda B, x, e_{0}, 1 / 2\right) .
$$

The uncountable family of positive numbers $\left(\delta_{\lambda}\right)_{\lambda \in \Lambda}$ cannot be summable, thus there exist $\lambda_{1}, \ldots, \lambda_{q}$ in $\Lambda$ such that $\sum_{i=1}^{q} \delta_{\lambda_{i}}>1$. Fix $\varepsilon>0$ with $(1-\varepsilon) \sum_{i=1}^{q} \delta_{\lambda_{i}}>$ 1. There exists $N_{0} \geq 0$ such that if $N \geq N_{0}$ and $i \in\{1, \ldots, q\}$, then

$$
\frac{1}{N} \#\left\{1 \leq n \leq N ;\left\|\lambda_{i}^{n} B^{n} x-e_{0}\right\|<1 / 2\right\}>(1-\varepsilon) \delta_{\lambda_{i}} .
$$

In particular, one can find $i_{1}$ and $i_{2}$ and infinitely many integers $n$ such that at the same time $n$ belongs to $A p p\left(\lambda_{i_{1}} B, x, e_{0}, 1 / 2\right)$ and $A p p\left(\lambda_{i_{2}} B, x, e_{0}, 1 / 2\right)$. Without loss of generality, one may suppose that $i_{1}=1, i_{2}=2$, and $\lambda_{2}>\lambda_{1}$. Let $r=\frac{\lambda_{2}}{\lambda_{1}}>1$, and choose $n$ large enough so that $r^{n}>3$. Now the following two implications are 
simultaneously true:

$$
\begin{aligned}
& \text { if }\left|\lambda_{1}^{n} x_{n}-1\right| \leq\left\|\left(\lambda_{1} B\right)^{n} x-e_{0}\right\| \leq 1 / 2, \text { then }\left|\lambda_{1}^{n} x_{n}\right| \geq \frac{1}{2}, \\
& \text { if }\left|\lambda_{2}^{n} x_{n}-1\right| \leq\left\|\left(\lambda_{2} B\right)^{n} x-e_{0}\right\| \leq 1 / 2, \text { then }\left|\lambda_{2}^{n} x_{n}\right| \leq \frac{3}{2} .
\end{aligned}
$$

This is clearly a contradiction, since $\left|\lambda_{2}^{n} x_{n}\right|=r^{n}\left|\lambda_{1}^{n} x_{n}\right|>3 / 2$.

The proof of Theorem 4.6 is inspired by the work of Costakis and Sambarino ([16]). It uses the following extension of the well-known result of Ansari ([2]) that any power $T^{q}, q \geq 1$, of $T$ is hypercyclic, and that moreover $T$ and $T^{q}$ have the same hypercyclic vectors. The same property holds for frequently hypercyclic operators.

Theorem 4.7. Let $T$ be a frequently hypercyclic operator on a Banach space $X$. For any $q \geq 1, T^{q}$ is frequently hypercyclic, and $T$ and $T^{q}$ share the same frequently hypercyclic vectors.

Proof. For simplicity's sake, we write down the proof for $q=2$, the general argument being exactly the same. Let $x$ be a frequently hypercyclic vector for $T, y$ any vector of $X$ and $\varepsilon>0$. Ansari's result [2] implies that $x$ and $T x$ are both hypercyclic for $T^{2}$, hence that there exist two integers $p_{0}$ and $q_{0}$ such that $\left\|T^{2 p_{0}} x-y\right\|<\frac{\varepsilon}{2}$ and $\left\|T^{2 q_{0}+1} x-y\right\|<\frac{\varepsilon}{2}$. Let

$$
E=\left\{n \in \mathbb{N} ;\left\|T^{n} x-x\right\|<\frac{\varepsilon}{2 \max \left(\left\|T^{2 p_{0}}\right\|,\left\|T^{2 q_{0}+1}\right\|\right)}\right\} .
$$

Then $E$ has positive lower density. Let $n$ belong to $E$. If $n$ is even and $n=2 m$, then

$$
\left\|T^{2\left(m+p_{0}\right)} x-y\right\| \leq\left\|T^{2 p_{0}}\left(T^{2 m} x-x\right)\right\|+\left\|T^{2 p_{0}} x-y\right\|<\varepsilon .
$$

If $n$ is odd and $n=2 m+1$, then

$$
\left\|T^{2\left(m+q_{0}+1\right)} x-y\right\| \leq\left\|T^{2 q_{0}+1}\left(T^{2 m+1} x-x\right)\right\|+\left\|T^{2 q_{0}+1} x-y\right\|<\varepsilon .
$$

Let $F=\left\{m+p_{0}, 2 m \in E\right\} \cup\left\{m+q_{0}+1,2 m+1 \in E\right\}$. We have to show that $F$ has positive lower density. But this follows from the fact that if $\# E \cap\{1,2, \ldots, N\} \geq$ $\delta N$, then either $\# E \cap\left\{2,4, \ldots, 2\left\lfloor\frac{N}{2}\right\rfloor\right\} \geq \frac{\delta}{2} N$ or $\# E \cap\left\{1,3, \ldots, 2\left\lfloor\frac{N-1}{2}\right\rfloor+1\right\} \geq$ $\frac{\delta}{2} N$. In the first case $\# F \cap\left\{1,2, \ldots,\left\lfloor\frac{N}{2}\right\rfloor+p_{0}\right\} \geq \frac{\delta}{2} N$, and in the second case $\# F \cap\left\{1,2, \ldots,\left\lfloor\frac{N}{2}\right\rfloor+q_{0}+1\right\} \geq \frac{\delta}{2} N$. Hence $F$ has positive lower density, and this finishes the proof.

We can now give the proof of Theorem 4.6.

Proof of Theorem 4.6. By Theorem 2.5, there exists a frequently hypercyclic vector $f$ for $T_{1}$. We claim that $f$ belongs to $F H C\left(T_{a}\right)$ for every $a>0$. Suppose first that $a$ is a rational number and write it as $p / q$. Fix $h \in \mathcal{H}(\mathbb{C}), K$ a compact subset of $\mathbb{C}$, and $\varepsilon>0$. Theorem 4.7 implies that $f$ is frequently hypercyclic for $T^{p}$, and it is straightforward to check that if $m$ satisfies $\left\|\left(T_{1}^{p}\right)^{m} f-h\right\|_{C(K)}<\varepsilon$, then $m q$ satisfies

$$
\left\|\left(T_{a}\right)^{m q} f-h\right\|_{C(K)}<\varepsilon
$$

which proves that $f$ belongs to $F H C\left(T_{a}\right)$. Suppose now that $a$ is irrational. Consider $h, K, \varepsilon$ as previously. The uniform continuity of $h$ on compact subsets of $\mathbb{C}$ 
implies the existence of a $\delta, 0<\delta<1$, such that $|h(z)-h(w)|<\varepsilon$ as soon as $z \in K$ and $|z-w|<\delta$. We set

$$
K_{\delta}=K+\delta \overline{\mathbb{D}},
$$

and choose $Q \geq 1$ an integer with $Q>2 \max _{z \in K_{\delta}}|z|$. The minimality of the rotation of irrational angle $a / Q$ (see [18) implies that there exist $M>0$ and an increasing sequence of integers $\left(n_{k}\right)$ such that

$$
\left\{\begin{array}{c}
n_{0}=0 \\
0 \leq n_{k} a[\bmod Q]<\delta, \\
n_{k+1}-n_{k}<M .
\end{array}\right.
$$

Writing $\alpha=\left[\frac{M a+1}{Q}\right]$, by Runge's Theorem there exists $h_{0} \in \mathcal{H}(\mathbb{C})$ satisfying

$$
\left|h_{0}(z)-h(z+j Q)\right|<\varepsilon
$$

for $z \in K_{\delta}-j Q, j=0, \ldots, \alpha$. We denote by $L$ the compact set

$$
L=K_{\delta} \cup\left(K_{\delta}-Q\right) \cup \cdots \cup\left(K_{\delta}-\alpha Q\right) .
$$

Since

$$
\operatorname{App}\left(T_{1}^{Q}, f, h_{0}, \varepsilon, L\right)=\left\{n \in \mathbb{N} ;\left\|\left(T_{1}^{Q}\right)^{N} f-h_{0}\right\|_{C(L)}<\varepsilon\right\}
$$

has positive lower density, one can find an increasing sequence of integers $\left(u_{k}\right)$ with positive lower density such that

$$
u_{k+1}-u_{k}>\frac{M a}{Q} \text { and } u_{k} \in A p p\left(T_{1}^{Q}, f, h_{0}, \varepsilon, L\right) .
$$

For each $k$, let $j(k)$ be the smallest possible integer such that $n_{j(k)} a \leq Q u_{k} \leq$ $n_{j(k)} a+M a$. Since $Q u_{k+1}>Q u_{k}+M a$ and $n_{k+1}-n_{k}<M$, it is plain that $n_{j(k)} \neq n_{j\left(k^{\prime}\right)}$ for $k \neq k^{\prime}$. Let us write $n_{j(k)} a=m_{k} Q+\delta_{k}$ with $0<\delta_{k}<\delta$, and $m_{k} Q=Q u_{k}-Q \alpha_{k}$. Since

$$
m_{k} Q \leq Q u_{k} \leq m_{k} Q+M a+1,
$$

one gets

$$
0 \leq \alpha_{k} \leq \frac{M a+1}{Q}
$$

Putting everything together, we obtain that for any $z$ in $K$,

$$
\begin{aligned}
\left|f\left(z+n_{j(k)} a\right)-h(z)\right| & =\left|f\left[\left(z+\delta_{k}-Q \alpha_{k}\right)+Q u_{k}\right]-h(z)\right| \\
& \leq \varepsilon+\left|h_{0}\left(z+\delta_{k}-Q \alpha_{k}\right)-h(z)\right| \\
& <2 \varepsilon+\left|h\left(z+\delta_{k}\right)-h(z)\right| \leq 3 \varepsilon .
\end{aligned}
$$

This proves that $n_{j(k)}$ belongs to $\operatorname{App}\left(T_{a}, f, h, 3 \varepsilon, K\right)$. Now, $u_{k} \mapsto n_{j(k)}$ is one-toone, and

$$
n_{j(k)} \leq \frac{Q u_{k}}{a} .
$$

The fact that $\underline{\operatorname{dens}}\left(u_{k}\right)>0$ ensures that $\underline{\operatorname{dens}}\left(\operatorname{App}\left(T_{a}, f, h, 3 \varepsilon, K\right)\right)>0$, and the claim follows.

In connection with the Hypercyclicity Criterion Problem, the following question is intriguing.

Question 4.8. Let $T$ be a frequently hypercyclic operator on $X$. Is $T \oplus T$ hypercyclic on $X \oplus X$ ? 
The answer is clearly affirmative for all the operators constructed here. Another question could be:

Question 4.9. Let $T$ be a frequently hypercyclic operator on $X$. Is $T \oplus T$ frequently hypercyclic on $X \oplus X$ ?

Again this is true for all the frequently hypercyclic operators exhibited here (if $T$ satisfies the assumptions of Theorem 2.1, so does $T \oplus T$, and if $T$ has a perfectly spanning set of eigenvectors associated to unimodular eigenvalues, so does $T \oplus T)$. But the question in the general setting does not seem to be trivial, because very little is known regarding the size of $F H C(T)$ : for all we know, $F H C(T)$ may be equal to $\mathbb{C}[T]\left(x_{0}\right) \backslash\{0\}$, where $x_{0}$ is a frequently hypercyclic vector for $T$, and of course in this case $T \oplus T$ cannot be frequently hypercyclic (a vector of the form $x \oplus p(T) x$ cannot even be hypercyclic: its orbit under the action of $T \oplus T$ is contained in the proper closed subspace of vectors $x_{1} \oplus x_{2}$ satisfying $\left.p(T) x_{1}-x_{2}=0\right)$. This possibility is ruled out in the hypercyclicity setting because $H C(T)$, being a dense $G_{\delta}$ set, cannot coincide with a $K_{\sigma}$ set.

We finish this paper with a last natural question concerning the existence of frequently hypercyclic operators on general separable Banach spaces. A result of Ansari ([3]) and Bernal-González ([7]) is that every separable Banach space of infinite dimension supports a hypercyclic operator.

Question 4.10. Does every separable Banach space of infinite dimension support a frequently hypercyclic operator?

A positive answer to Question 4.10 would request that we construct frequently hypercyclic operators of the form $T=I+Q$, where $Q$ is a compact quasinilpotent operator, but we do not know whether this is possible or not (we rather suspect it is not ...).

\section{ACKNOWLEDGEMENT}

We are grateful to the referee for many valuable suggestions which greatly improved the presentation of this paper.

\section{ADDED IN PROOF}

Conjecture 2.10 has been disproved by K.-G. Grosse-Erdmann and A. Peris in Frequently dense orbits, C. R. Math. Acad. Sci. Paris 341 (2005), pp 123-128, MR2153969 (2006a:47017). In this same paper, the authors give a positive answer to Question 4.8 .

\section{REFERENCES}

[1] E. Abakumov, J. Gordon, Common hypercyclic vectors for multiples of the backward shift, J. Funct. Anal., 200 (2003), pp 494 - 504. MR.1979020 (2004g:47012)

[2] S.I. Ansari, Hypercyclic and cyclic vectors, J. Funct. Anal., 128 (1995), pp $374-383$. MR1319961 (96h:47002)

[3] S.I. ANSARI, Existence of hypercyclic operators on topological vector spaces, J. Funct. Anal., 148 (1997), pp 384 - 390. MR:1469346(98h:47028a)

[4] F. Bayart, S. Grivaux, Hypercyclicity: the role of the unimodular point spectrum, C. R. Acad. Sci. Paris, 338 (2004), pp 703 - 708. MR2065378 (2005c:47009)

[5] F. Bayart, S. Grivaux, Hypercyclicity and unimodular point spectrum, J. Funct. Anal., 226 (2005), pp 281-300. MR2159459 
[6] F. Bayart, S. Grivaux, Invariant gaussian measures for operators on Banach spaces and linear dynamics, to appear in Proc. London Math. Soc.

[7] L. Bernal-GonzÁlez, On hypercyclic operators on Banach spaces, Proc. Amer. Math. Soc., 127 (1999), pp 1003 - 1010. MR.1476119 (99f:47010)

[8] T. Bermudez, A. Bonilla, A. Peris, On Hypercyclicity and Supercyclicity Criteria, Bull. Austral. Math. Soc., 70 (2004), pp 45-54. MR2079359 (2005d:47014)

[9] J. Bìs, Invariant linear subspaces of hypercyclic vectors for the real scalar case, Proc. Amer. Math. Soc., 127 (1999), pp 1801 - 1804. MR1485460(99i:47002)

[10] J. Bès, A. PerIs, Hereditarily hypercyclic operators, J. Funct. Anal., 167 (1999), pp 94 112. MR 1710637 (2000f:47012)

[11] G. D. Birkhoff, Démonstration d'un théorème élémentaire sur les fonctions entières, $C$. $R$. Acad. Sci. Paris, 189 (1929), pp 473 - 475.

[12] V. I. Bogachev, Gaussian measures, Mathematical Surveys and Monographs, 62, American Mathematical Society, Providence, RI, 1998. MR1642391 (2000a:60004)

[13] P. Bourdon, Invariant manifolds of hypercyclic vectors, Proc. Amer. Math. Soc., 118 (1993), pp 845 - 847. MR1148021(93i:47002)

[14] P. Bourdon, J. H. Shapiro, Hypercyclic operators that commute with the Bergman backward shift, Trans. Amer. Math. Soc., 352 (2000), pp 5293 - 5316. MR1778507(2001i:47053)

[15] P. Bourdon, J. H. Shapiro, Cyclic phenomena for composition operators, Mem. Amer. Math. Soc., 125 (1997), no 596. 125 (1997), no. 596, x+105 pp. MR1396955 (97h:47023)

[16] G. Costakis, M. Sambarino, Genericity of wild holomorphic functions and common hypercyclic vectors, Adv. Math., 182 (2004), pp 278 - 306. MR2032030 (2004k:47009)

[17] J. Dixmier, C. Foias, Sur le spectre ponctuel d'un opérateur, Coll. Math. Soc. Janos Bolyai, Tihany (Hungary) (1970), pp 127 - 133. MR0365175 (51:1428)

[18] R. Ellis, Lectures on topological dynamics, W.A. Benjamin, Inc., New York, 1969. MR0267561 (42:2463)

[19] N. FELDMAN, Linear Chaos, http://home.wlu.edu/ feldmann/research.html.

[20] E. Flytzanis, L. Kanakis, Unimodular eigenvalues and univariant probabilities for some classes of linear operators, Probability theory and mathematical statistics, Proceedings of the sixth Vilnius conference, Vilnius, Lithuania (1993), pp 277 - 283. MR.1649581 (99g:47014)

[21] E. Flytzanis, Unimodular eigenvalues and linear chaos in Hilbert spaces, Geom. Funct. Analysis, 5 (1995), pp 1 - 13. MR1312018 (95k:28034)

[22] E. Flytzanis, Mixing properties of linear operators in Hilbert spaces, Choquet, G. (ed.) et al., Séminaire d'initiation à l'analyse, 34ème année: 1994/1995, Publ. Math. Univ. Pierre Marie Curie, Exp. No. 6, 117 (1995).

[23] D. Gaier, Lectures on complex approximation, Birkhauser Boston, Inc., Boston, MA, 1987. MR0894920 (88i:30059b)

[24] E. Gallardo-Gutiérrez, A. Montes-Rodríguez, Role of the spectrum in the cyclic behavior of composition operators, Memoirs of the American Mathematical Society, 791 (2004). MR2023381 (2004k:47050)

[25] E. Gallardo-Gutiérrez, A. Montes-Rodríguez, The role of the angle in supercyclic behaviour, J. Funct. Anal, 203 (2003), pp 27 - 43. MR1996867(2004h:47012)

[26] R. Gethner and J. H. Shapiro, Universal vectors for operators on spaces of holomorphic functions, Proc. Amer. Math. Soc., 100 (1987), pp 281 - 288. MR0884467 (88g:47060)

[27] G. Godefroy, J. H. Shapiro, Operators with dense, invariant, cyclic vector manifolds, J. Funct. Anal., 98 (1991), pp 229 - 269. MR1111569 (92d:47029)

[28] S. Grivaux, Construction of operators with prescribed behaviour, Archiv der Math., 81 (2003), pp 291 - 299. MR2013260 (2004g:47011)

[29] S. Grivaux, Sums of hypercyclic operators, J. Funct. Anal., 202 (2003), pp 486 - 503. MR.1990535 (2004c:47017)

[30] S. GrivaUX, Topologically transitive extensions of bounded operators, Math. Z., 249 (2005), pp 85 - 96. MR2106971 (2005g:47008)

[31] S. Grivaux, Hypercyclic operators, mixing operators, and the bounded steps problem, J. Operator Th., 54 (2005), no. 1, pp 147-168. MR2168865

[32] K-G. Grosse-Erdmann, Universal families and hypercyclic operators, Bull. Amer. Math. Soc., 36 (1999), pp 345 - 381. MR.1685272 (2000c:47001)

[33] K-G. Grosse-Erdmann, Recent developments in hypercyclicity, RACSAM Rev. R. Acad. Cienc. Exactas Fis. Nat. Ser. A Mat. 97 (2003), pp 273 - 286. MR2068180(2005c:47010) 
[34] D. A. Herrero, Hypercyclic operators and chaos, J. Operator Theory, 28 (1992), pp 93 103. MR.1259918 (95g:47031)

[35] S. Janson, Gaussian Hilbert spaces, Cambridge Tracts in Mathematics, 129, Cambridge University Press, Cambridge, 1997. MR.1474726 (99f:60082)

[36] Y. Katznelson, An Introduction to Harmonic Analysis, John Wiley \& Sons, New York, London, Sydney, 1968. MR0248482(40:1734)

[37] A. Kechris, A. Louveau, Descriptive set theory and the structure of sets of uniqueness, London Mathematical Society Lecture Note Series, 128, Cambridge University Press, Cambridge, 1987. MR0953784 (90a:42008)

[38] C. KitAI, Invariant closed sets for linear operators, Ph.D. thesis, Univ. of Toronto, 1982.

[39] H. H. Kuo, Gaussian measures in Banach spaces, Lecture Notes in Mathematics, 463, Springer-Verlag, 1975. MR0461643 (57:1628)

[40] G. R. MacLane, Sequences of derivatives and normal families, J. Analyse. Math., 2 (1952), pp 72 - 87. MR0053231 (14:741d)

[41] V. Müller, Local spectral radius formula for operators in Banach spaces, Czech. Math. Journal, 38 (1988), pp 726 - 729. MR0962915 (89g:47005)

[42] L. Nikolskaya, Geometric properties of system of characteristic vectors and point spectra of linear operators, Fun. Ann. Appl., 4 (1970), pp $105-106$.

[43] L. NikOlSkAYA, Structure of the point spectrum of a linear operator, Mat. Zametki, 15 (1974), pp 149 - 158, English transl. in Math. Notes, 15 (1974). MR0346554 (49:11279)

[44] E. Nordgren, P. Rosenthal, F. S. Wintrobe, Invertible composition operators on $H^{p}, J$. Funct. Anal., 73 (1987), pp 324 - 344. MR0899654 (89c:47044)

[45] V. Peller, Hankel operators and their applications, Springer Monographs in Mathematics, Springer-Verlag, New York, 2003. MR.1949210 (2004e:47040)

[46] S. Rolewicz, On orbits of elements, Studia Math., 32 (1969), pp 17 - 22. MR0241956 $(39: 3292)$

[47] H. Salas, Hypercyclic weighted shifts, Trans. Amer. Math. Soc., 347 (1995), pp $993-1004$. MR.1249890 (95e:47042)

[48] H. Salas, Supercyclicity and weighted shifts, Sudia Math., 135 (1999), pp 55 - 74. MR1686371 (2000b:47020)

[49] J. H. Shapiro, Decomposability and the cyclic behavior of parabolic composition operators, Recent progresses in functional analysis (Valencia 2000), pp $143-157$, North-Holland Math. Stud., 189, North-Holland, Amsterdam, 2001. MR.1861753 (2002g:47049)

[50] P. Walters, An Introduction to Ergodic Theory, Graduate Texts in Mathematics, 79, Springer-Verlag, New York, Berlin, 1982. MR0648108 (84e:28017)

[51] J. Wengenroth, Hypercyclic operators on non-locally convex spaces, Proc. Amer. Math. Soc., 131 (2003), pp 1759 - 1761. MR1955262(2003j:47007)

Laboratoire Bordelais d'Analyse et de Géométrie, UMR 5467, Université Bordeaux 1, 351 Cours de la libération, 33405 Talence Cedex, France

E-mail address: bayart@math.u-bordeaux.fr

Laboratoire Paul Painlevé, UMR 8524, Université des Sciences et Technologies de Lille, Cité Scientifique, 59655 Villeneuve d'Ascq Cedex, France

E-mail address: grivaux@math.univ-lille1.fr 